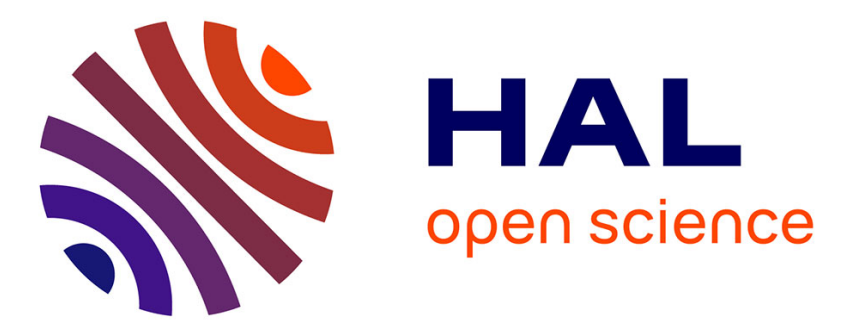

\title{
Thermal-field effects on interface dynamics and microstructure selection during alloy directional solidification
}

Y. Song, D. Tourret, F.L. L Mota, J. Pereda, B. Billia, N. Bergeon, R. Trivedi, A. Karma

\section{To cite this version:}

Y. Song, D. Tourret, F.L. L Mota, J. Pereda, B. Billia, et al.. Thermal-field effects on interface dynamics and microstructure selection during alloy directional solidification. Acta Materialia, 2018, 150, pp.139-152. 10.1016/j.actamat.2018.03.012 . hal-01785653

\section{HAL Id: hal-01785653 \\ https://hal.science/hal-01785653}

Submitted on 24 May 2018

HAL is a multi-disciplinary open access archive for the deposit and dissemination of scientific research documents, whether they are published or not. The documents may come from teaching and research institutions in France or abroad, or from public or private research centers.
L'archive ouverte pluridisciplinaire HAL, est destinée au dépôt et à la diffusion de documents scientifiques de niveau recherche, publiés ou non, émanant des établissements d'enseignement et de recherche français ou étrangers, des laboratoires publics ou privés. 


\section{Accepted Manuscript}

Thermal-field effects on interface dynamics and microstructure selection during alloy directional solidification

Y. Song, D. Tourret, F.L. Mota, J. Pereda, B. Billia, N. Bergeon, R. Trivedi, A. Karma

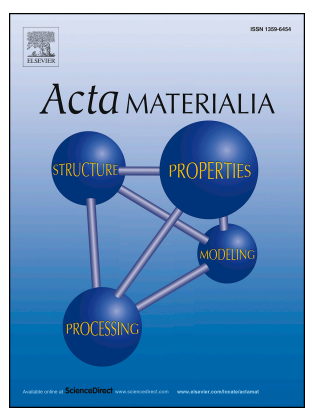

PII: S1359-6454(18)30191-5

DOI: $\quad$ 10.1016/j.actamat.2018.03.012

Reference: $\quad$ AM 14430

To appear in: Acta Materialia

Received Date: 3 January 2018

Revised Date: 28 February 2018

Accepted Date: 3 March 2018

Please cite this article as: Y. Song, D. Tourret, F.L. Mota, J. Pereda, B. Billia, N. Bergeon, R. Trivedi, A. Karma, Thermal-field effects on interface dynamics and microstructure selection during alloy directional solidification, Acta Materialia (2018), doi: 10.1016/j.actamat.2018.03.012.

This is a PDF file of an unedited manuscript that has been accepted for publication. As a service to our customers we are providing this early version of the manuscript. The manuscript will undergo copyediting, typesetting, and review of the resulting proof before it is published in its final form. Please note that during the production process errors may be discovered which could affect the content, and all legal disclaimers that apply to the journal pertain. 


\title{
Thermal-field effects on interface dynamics and microstructure selection during alloy directional solidification
}

\author{
Y. Song ${ }^{1}$, D. Tourret ${ }^{2}$, F.L. Mota $^{3}$, J. Pereda ${ }^{3}$, B. Billia ${ }^{3}$, N. Bergeon ${ }^{3}$, R. Trivedi ${ }^{4}$, A. Karma ${ }^{1 *}$ \\ ${ }^{1}$ Department of Physics and Center for Interdisciplinary Research on Complex Systems, \\ Northeastern University, Boston, MA, USA \\ ${ }^{2}$ IMDEA Materials Institute, Getafe, Madrid, Spain \\ ${ }^{3}$ Institut Matériaux Microélectronique Nanosciences de Provence, Aix-Marseille Université and CNRS, Marseille, France \\ ${ }^{4}$ Department of Material Science and Engineering, Iowa State University, Ames, IA, USA
}

\begin{abstract}
We carry out three-dimensional phase-field simulations to model unique experimental observations of cellular and dendritic solidification structures formed under diffusive growth conditions in the DSI (Directional Solidification Insert) of the DECLIC (DEvice for the study of Critical LIquids and Crystallization) aboard the International Space Station. We had previously shown experimentally that complex thermal conditions affect the stationary position of the solid-liquid interface, as well as its dynamics of relaxation towards this stationary position over a finite time after the onset of sample pulling. Here, we discuss the effects of thermal diffusion within the adiabatic zone of the directional solidification setup and of latent heat release at the solid-liquid interface by means of quantitative phase-field simulations. Simulations and experiments characterize the entire evolution of the primary spacing of cellular/dendritic array structures from the onset of morphological instability to the establishment of the final steady-state spacing, including the transient coarsening regime associated with a sharp increase of spacing. Accounting for these thermal effects leads to a major improvement in the agreement between simulations and microgravity measurements for both the time of occurrence of morphological instability after the start of the experiment and the subsequent spacing evolution, which are not accurately predicted using the standard frozen temperature approximation.
\end{abstract}

Keywords: Directional solidification, Phase field, Microstructure formation, Alloys

\section{Introduction}

Columnar microstructures form when a liquid material solidifies under a temperature gradient. Resulting spacings between primary or secondary branches in these microstructures affect their mechanical proper-

* Corresponding author

Email address: a.karma@northeastern.edu 
ties $[1,2,3]$. In order to explore a variety of columnar microstructures, experiments have been carried out for several decades with the Bridgman method [4].

On Earth, gravity leads to solutal and thermal convective currents in the liquid phase, which yield inhomogeneous microstructures [5, 6]. Thus, solidification experiments on Earth have primarily been performed with a thin-sample geometry $[7,8,9,10,11,12,13,14,15,16]$, in order to reduce convection and operate in a mostly diffusive regime. For bulk three-dimensional (3D) experiments, reduced gravity conditions are necessary to investigate homogeneous microstructure growth, which has motivated solidification experiments in Space for over twenty years $[17,18,19]$.

Recently, directional solidification experiments on a transparent organic compound were performed in the Directional Solidification Insert (DSI) of the DEvice for the study of Critical LIquids and Crystallization (DECLIC) aboard the International Space Station (ISS). These microgravity experiments revealed a wide range of complex 3D microstructure dynamics under various experimental conditions [19]. Especially, the experimental observations highlighted oscillatory modes in cellular structures over a finite range of growth conditions, which were investigated by 3D phase-field (PF) simulations [20, 21, 22]. Yet, while PF simulations accurately reproduced the oscillation characteristics and identified the origin of these oscillations as linked to a spacing stability limit $[20,21]$, they had so far fallen short of accurately predicting primary spacings, systematically smaller in simulations than in experiments. In addition, simulations were limited to a low velocity range and focused on the final steady-state spacing.

Mechanisms of dynamical spacing evolution have been extensively studied analytically and experimentally, mostly in a thin-sample geometry $[7,8,9,10,11,12,13,14,15,16]$. Classical models describe primary spacing selection with a combination of power laws of the freezing range of the alloy, pulling velocity, and temperature gradient $[8,7,11,16]$. However, even though similar processing history tends to yield similar selected spacings $[9,10,12,13]$, pattern stability was shown over a wide range of spacings for a given set of control parameters $[8,9,12,13,14,16,23,24,25,26]$. The lower limit of spacing stability $\Lambda_{\min }$ is linked to an elimination instability, while its upper limit $\Lambda_{\max }$ relates to a tertiary branching instability (or tip-splitting instability in a cellular growth regime).

Hunt and Lu first mentioned that, in order for the branching instability to results in a stable spacing with respect to elimination, $\Lambda_{\max }$ had to be at least $2 \Lambda_{\min }[24,25]$. Because there was no way to calculate $\Lambda_{\max }$ at the time, they suggested that $\Lambda_{\max } \approx 2 \Lambda_{\min }$, which yields good agreement with most experimental data. More recent studies using both phase-field and a multiscale model for dendritic growth have shown that the ratio $\Lambda_{\max } / \Lambda_{\min }$ may be closer to 3 or 4 [26, 27, 28, 29]. However, dynamically selected spacings in spatially extended domains were found to be mostly distributed within the lower half of the stability range, hence between $\Lambda_{\min }$ and $2 \Lambda_{\min }$.

Because of the impossibility to process homogeneous bulk samples due to gravity-driven buoyancy on Earth, the available experimental data on microstructure selection under well-controlled conditions has been 
limited to quasi two-dimensional thin-sample experiments. The DECLIC-DSI experiments provide the very first set of experimental data on 3D microstructure formation, evolution, and selection in a predominantly diffusive transport regime. Those experiments provide an unprecedented opportunity to visualize the entire history-dependent selection of cellular/dendritic microstructures from the initial breakdown of the planar interface to the establishment of a steady-state primary spacing.

Recently, a thorough analysis of the initial recoil of the planar solid-liquid interface within the temperature field, as the sample is pulled towards the colder region from its initial liquidus position, shed light on complex thermal conditions within the experimental setup [30]. In order to properly reproduce the transient interface dynamics, one needs to account for two thermal effects: (i) finite heat transport inside the sample within the adiabatic zone, and (ii) latent heat rejection as the liquid solidifies. These two factors were initially not considered in the classical frozen temperature approximation within our PF simulations.

In the present article, we characterize the entire evolution of the primary spacing of cellular/dendritic array structures that starts with the initial breakdown of the planar interface and is followed by the growth competition of finger shaped cells, which leads to a rapid increase of spacing and the establishment of the dynamically selected steady-state spacing. While other effects can influence the evolution of the primary spacing even after the solid-liquid interface has reached a steady-state velocity (such as sub-grain boundaries or the macroscopic curvature of the solid-liquid interface on the sample scale), we focus in the present article on characterizing the history-dependent selection of the spacing for a single grain and a macroscopically flat solidification front.

We show that a much better agreement between measured and simulated interface dynamics and microstructure selection can be achieved by (i) using a carefully reevaluated solute partition coefficient at the solid-liquid interface [31], and (ii) accounting for non-ideal thermal conditions within the experimental setup [30]. To do so, we explore different representations of the thermal field within PF simulations. Namely, we compare: (i) the classical frozen temperature approximation, (ii) a phenomenological introduction of the thermal drift adjusted to experimental measurements, and (iii) a time-dependent calculation of the temperature field within the sample coupled with PF simulations. By comparing quantitatively simulations and experiments, we find that non-ideal thermal conditions, linked to latent heat release and finite thermal transport, have a profound effect on the history-dependent microstructure selection in 3D samples.

\section{Microgravity experiments}

Experiments discussed here were performed in the DSI of the DECLIC developed by the French space agency (CNES) and installed onboard the ISS [32, 33, 34]. The DECLIC-DSI setup is dedicated to in situ and real time characterization of the solid-liquid interface dynamics in 3D bulk samples of transparent materials. 
Within the DSI, a cylindrical crucible of inner diameter of $10 \mathrm{~mm}$ is solidified over a length of up to $10 \mathrm{~cm}$ by pulling it through an adiabatic zone located between hot and cold zones of a Bridgman furnace (upper figure in Fig. 1). A flat glass window at the bottom of the crucible and an immersed lens at the top (i.e. in the hot zone) allow direct axial imaging of the interface. Transparency of the crucible also permits transversal imaging, thus providing both side view and top view images of the solidifying microstructure. More details on the DECLIC-DSI setup and experiments can be found elsewhere [19, 22, 30, 32, 33, 34, 35, 36].

The first spatial campaign of DECLIC-DSI experiments was performed between April 2010 and February 2011 on a succinonitrile (SCN)-0.24wt\% camphor alloy, for two different temperature gradients $G$ and a wide range of pulling velocities $V[19,30]$. In this article, we focus on experiments performed at $G=19 \mathrm{~K} / \mathrm{cm}$ and velocities from $V=1$ to $8 \mu \mathrm{m} / \mathrm{s}$.

Fig. 2 illustrates the evolution of the solid-liquid interface during the directional solidification experiment for $V=4 \mu \mathrm{m} / \mathrm{s}$. Microstructure development and evolution at other velocities typically proceed similarly as in Fig. 2.

Experiments start with planar solid-liquid interface at rest close to the location of the alloy liquidus temperature (i.e. $330.85 \mathrm{~K}$ for a $\mathrm{SCN}-0.24 \mathrm{wt} \%$ camphor alloy) within the imposed temperature gradient (Fig. 2a). When the sample is pulled towards the colder region of the apparatus, the interface initially grows slower than the pulling velocity, such that it recoils within the temperature gradient. The liquid solute supersaturation thus increases, until the planar interface becomes morphologically unstable [38, 39]. At the onset of morphological instability, ridges and poxes start forming on the interface as illustrated in Fig. 2b [19, 34, 37]. The uniform corrugation progressively develops, which corresponds to the initial visible wavelength of morphological instability (Fig. 2c). This initial perturbation grows to form small cells that compete with one another, causing the pattern to select a primary spacing, mainly through solutal interactions among cells or dendrites. Then, the microstructure approaches a stationary state as time elapses (Fig. 2d-f).

Fig. 3a illustrates the detailed evolutions of average primary spacing $\Lambda$ at $V[\mu \mathrm{m}]=1$ (gray down triangles), 2 (red squares), 4 (green circles), and 8 (blue up triangles) as a function of the solidified length $L=V t$. Primary spacings are calculated in a similar fashion as presented in Ref. [21], in both experiments and simulations, i.e. using a Voronoi tessellation of space and extracting individual center-to-center distances between all neighbor cells. However, at the early stages of microstructure formation, it is not possible to determine the spacing with center-to-center methods because the grooves between primary cells are not deep enough. Therefore, the initial estimation of the average $\Lambda$ is performed using fast Fourier transform (FFT) analysis (Fig. 2c). The FFT analysis only pertains to the early stage, i.e. at most the first two data points in Fig. 3a, and is found to provide a good continuity and overlap with the more accurate centerto-center analysis where both methods are applicable. As shown in Fig. 2c-f (black crosses in Fig. 3a), 3D microgravity experiments for $V \geq 2 \mu \mathrm{m} / \mathrm{s}$ in Fig. 3a typically exhibit a peak spacing value before it 
slowly decreases towards a steady state value. A similar microstructure evolution was reported in previous experiments with a thin-sample geometry [40]. At low velocities $V \leq 1 \mu \mathrm{m} / \mathrm{s}$, the primary spacing typically increases monotonically towards its steady state value.

Fig. 3b shows the distribution of cell spacing across the interface in the steady state for $V=4 \mu \mathrm{m} / \mathrm{s}$ (corresponding to Fig. 2f). A small number of spacings appear higher, which we attribute to the presence of a subgrain boundary on the right-hand side of the image. This boundary appears with a clear difference of luminosity between the two regions in Fig. 2e-f. The macroscopic curvature of the solidification front, linked to an inhomogeneity of the radial temperature field, also contributes to local pattern drifting and local heterogeneities of spacings, as already discussed in Ref. [22]. However, in spite of some outliers, the spacing standard deviation $s_{\Lambda}=24.7 \mu \mathrm{m}$ remains low compared to its mean value $\Lambda=185 \mu \mathrm{m}$. This illustrates the remarkably uniform microstructure distribution at a stationary state that can only be obtained in bulk samples under reduced gravity conditions.

\section{Phase-field modeling}

\subsection{Phase-field model}

The phase-field (PF) approach has emerged over the past decades as the method of choice to explore a variety of complex microstructure dynamics [41, 42, 43, 44]. The quantitative phase-field (PF) model of solidification used here takes advantage of a thin-interface asymptotic analysis, which allows to remain accurate with a numerical diffuse interface width much larger than the actual physical interface $[45,46]$. The model neglects solid-state diffusion and is made quantitatively accurate by using an anti-trapping solute flux that corrects the spurious solute trapping effects appearing when using such a wide diffuse interface [47, 48].

The model equations are solved using finite differences on a cubic grid and an explicit time stepping scheme. Computational efficiency was improved by: (i) solving the model equations for a preconditioned phase field $\psi$ instead of the usual phase field $\varphi$ (with $\varphi=-1$ in the liquid and +1 in the solid) through the nonlinear change of variable $\varphi \equiv \tanh (\psi / \sqrt{2})$, which enhances stability for larger grid spacings [21, 49]; and (ii) implementing the model on massively parallel GPU (Graphics Processing Unit) architectures using Nvidia's CUDA programing platform [50].

This model was already used extensively to study directional solidification experiments $[20,21,22,51$, $52,26,53,54]$. It was shown to provide good agreements with solidification experiments in transparent alloys $[26,53,54]$, and especially reproducing quantitative features of oscillatory dynamics observed during cellular growth in DECLIC-DSI experiments [20, 21, 22].

\subsection{Numerical description of the thermal field}

Recently, we discussed the appropriate description of the thermal field within the experimental setup based on experimental measurements of the interface position and its time evolution during the early stage 
of the experiment when the interface is still close to planar [30]. In the current article, we use 3D PF simulations to compare different descriptions of the thermal field, and discuss their validity in light of the measured interface transient dynamics, and selected microstructures. Namely, we compare three distinct modeling approaches (briefly described in the following subsections):

- The standard Frozen Temperature Approximation (FTA), assuming a fixed homogeneous temperature gradient moving at a given constant velocity;

- A recently proposed Thermal Drift Approximation (TDA), with a fixed temperature gradient experiencing a shift with time that is adjusted from experimental measurements [30];

- A time-dependent Thermal Field Calculation (TFC), which couples the phase-field model to a timedependent calculation of thermal diffusion in the adiabatic zone.

For the latter, we consider a one-dimensional (1D) temperature field in most simulations throughout the article. For spatially extended simulations, we also perform TFC simulations with a full three-dimensional (3D) temperature field.

\subsubsection{Frozen Temperature Approximation}

Thermal diffusion being usually much faster than solute diffusion, it is often reasonable to assume that the temperature field is fixed with a given temperature gradient $G$. Thus, in the lab frame, the temperature $T$ along the $z$ axis is

$$
T=T_{0}+G z
$$

where $T_{0}$ is the solidus temperature of the alloy, taken as reference at $z=0$.

This Frozen Temperature Approximation (FTA), which yields equations summarized in Appendix A, is commonly used in phase-field calculations $[20,21,22,51,52,26,53,54]$. While it is usually valid for experiments on metals and metallic alloys, typically exhibiting at least four orders of magnitude of difference between solutal and thermal diffusivities, this might not always be appropriate for organic compounds such as SCN-based alloys. In addition, because the thermal diffusivity of a sample may be smaller than that of its surrounding crucible, the thermal field between heat sources inside a 3D sample may differ further from the FTA.

\subsubsection{Thermal Drift Approximation}

In a previous study [30], we demonstrated that, in the DECLIC-DSI experimental setup with a bulk cylindrical crucible of an organic compound, heat diffusion inside the crucible and latent heat rejection at the solid-liquid interface were responsible for a thermal drift of the isotherms. We proposed a modification 
of the FTA that phenomenologically describes this thermal drift, introducing a total isotherm shift $\Delta z_{T}$ and a delay time $\tau_{d}$, yielding the Thermal Drift Approximation (TDA)

$$
T=T_{0}+G z+G \Delta z_{T}\left(1-e^{-t / \tau_{d}}\right) .
$$

The total isotherm shift is estimated from the experimentally measured interface recoil $\Delta z_{\text {exp }}$ with $\Delta z_{T}=\Delta z_{\text {exp }}-\Delta \cdot l_{T}$, where $\Delta$ is the dimensionless microstructure tip undercooling (actual undercooling divided by the freezing range), and $l_{T}$ is the alloy thermal length (i.e. the distance between solidus and liquidus temperatures at its nominal composition). The characteristic relaxation time for the thermal field $\tau_{d}$ can be estimated using a modified version of the classical Warren-Langer (WL) model [30, 23]. The undercooling $\Delta$ can be estimated using analytical expressions, such as those discussed in Sec. 4.4, or using $\mathrm{PF}$, as done here with simulations of a subset of the experiment, namely one quarter of a cell (see Sec. 3.3). Further details of the TDA equations are in Appendix B.

\subsubsection{Thermal Field Calculation}

Having identified the two sources of the thermal drift, namely finite heat diffusion and latent heat release, we can quantitatively calculate the evolution of the temperature field within the adiabatic zone between heat sources.

To keep simulations computationally tractable, we typically consider the time evolution of a onedimensional (1D) temperature field along the $z$ direction, following

$$
\partial_{t} T=V \partial_{z} T+D_{T} \partial_{z z} T+\frac{\Delta h_{f}}{c_{p}} \frac{1}{\mathcal{V}} \int \frac{\dot{\varphi}}{2} \mathrm{~d} \mathcal{V},
$$

where $D_{T}$ is the thermal diffusivity, $\Delta h_{f}$ is the alloy latent heat of fusion per unit volume, and $c_{p}$ is its heat capacity.

Like for the phase and solute fields, we solve Eq. (3) using finite differences and explicit Euler time stepping. We use a same time step $\Delta t$ for all equations. Hence, since solute and heat diffusivities, respectively $D$ and $D_{T}$, are orders of magnitude apart, with $D_{T} \gg D$, we solve Eq. (3) on a grid spacing $\Delta x_{T}$ that is coarser than $\Delta x$ on which the phase and solute fields are calculated. In Eq. (3), $\mathrm{d} \mathcal{V}$ is used as short-hand notation to denote summation of the integrand over the points of the finer grid within a range of one $\Delta x_{T}$. Thus, the last term for the latent heat rejection is numerically integrated over a volume $\mathcal{V}=\Delta x_{T} \times L_{x} \times L_{y}$, with $L_{x}$ and $L_{y}$ the total domain size respectively in $x$ and $y$.

Spatially extended simulations were also performed using a full 3D temperature field calculation. The evolution of the 3D field follows

$$
\partial_{t} T=V \partial_{z} T+D_{T} \nabla^{2} T+\frac{\Delta h_{f}}{c_{p}} \frac{1}{\mathcal{V}} \int \frac{\dot{\varphi}}{2} \mathrm{~d} \mathcal{V} .
$$

Since $D_{T} \gg D$, the grid spacing for solving the thermal problem, $\Delta x_{T}$, is coarser than the grid size for the solute and phase field, $\Delta x$, using a fixed time step $\Delta t$. For the latent heat calculations, we integrated 
the latent heat rejection over a volume $\mathcal{V}=\Delta x_{T}^{3}$. Further details for the temperature field calculation are explained in Appendix C.

Temperatures at both ends of the adiabatic zone are fixed. Hence, boundary conditions in $z$ on the temperature field are set as Dirichlet conditions with $T=T_{c}$ at the (cold) low $z$ boundary and $T=T_{h}$ at the (hot) high $z$ boundary. Temperatures $T_{c}$ and $T_{h}$ are estimated from 2D axisymmetric thermal simulations of the entire setup [30]. We assume the thermal diffusivity in the liquid and solid phases to be equal because they are close to each other and much larger than the solute diffusivity [2].

\subsection{Simulations and parameters}

For the SCN-0.24wt\% camphor alloy, we used similar parameters as in Ref. [21, 22], with a crystalline anisotropy $\varepsilon_{4}=0.011$.

In the TDA simulations, for each experiment we calculated $\Delta z_{T}$ using the dimensionless tip undercooling $\Delta$ predicted by the PF simulation of a quarter of a cell at the average spacing measured in the corresponding experiment (i.e. as plot in Fig. 8a discussed later). Using this value of $\Delta z_{T}, \tau_{d}$ was estimated by fitting experimental measurements of the interface position during its transient planar recoil with a modified Warren-Langer (WL) model [30, 23], using an interfacial solute partition coefficient $k=0.07$ [31]. Resulting thermal drift parameters are summarized in Table 1.

For the TFC, since we consider a dilute alloy, we used $\Delta h_{f} / c_{p}=23 \mathrm{~K}$ as for pure SCN $[2,55]$. The inner radius of the crucible is $5 \mathrm{~mm}$ and the wall is $1 \mathrm{~mm}$ thick. The thermal conductivity of the alloy is close to that of pure SCN, i.e. $\kappa_{S C N}=0.224 \mathrm{~W} / \mathrm{m} / \mathrm{K}[2,9]$, and that of the quartz crucible is $\kappa_{C}=1.42 \mathrm{~W} / \mathrm{m} / \mathrm{K}$. Then, the effective thermal conductivity is estimated as $\kappa_{E}=\left(\kappa_{S C N} A_{S C N}+\kappa_{C} A_{C}\right) / A_{t o t}=0.589 \mathrm{~W} / \mathrm{m} / \mathrm{K}$, where the cross section area inside the crucible $A_{S C N}$, that of the crucible wall is $A_{C}$, and the total $A_{t o t}=A_{S C N}+A_{C}$. In addition, the effective heat capacity is estimated as $c_{p}^{E}=\left(c_{p}^{S C N} A_{S C N}+c_{p}^{C} A_{C}\right) / A_{t o t}=$ $1.91 \times 10^{6} \mathrm{~J} / \mathrm{m}^{3} / \mathrm{K}$ with $c_{p}^{S C N}=2.0 \times 10^{6} \mathrm{~J} / \mathrm{m}^{3} / \mathrm{K}$ for the SCN $[2,55]$ and $c_{p}^{C}=1.7 \times 10^{6} \mathrm{~J} / \mathrm{m}^{3} / \mathrm{K}$ for the quartz. Therefore, the effective thermal diffusivity inside the crucible is $D_{T}=\kappa_{E} / c_{p}^{E}=3.09 \times 10^{-7} \mathrm{~m}^{2} / \mathrm{s}$.

Assuming that the initial field and the boundary temperatures at the locations of the heat sources are independent of the pulling velocity, we estimated $T_{h}$ and $T_{c}$ from axisymmetric thermal simulations of the experimental setup [30], using the axial temperature field at rest (i.e. $V=0 \mu \mathrm{m} / \mathrm{s}$ ). Resulting boundary conditions on the temperature in the TFC are $T_{h}=348.13 \mathrm{~K}$ and $T_{c}=304.63 \mathrm{~K}$ over an adiabatic zone length of $2.34 \mathrm{~cm}$ (Fig. 1). Accordingly, the field is initialized with a nominal temperature gradient $G=19 \mathrm{~K} / \mathrm{cm}$.

We numerically investigated four pulling velocities $V[\mu \mathrm{m} / \mathrm{s}]=1,2,4$, and 8. Keeping $\Delta x / W=1.2$ for all velocities, we used a diffuse interface width $W / d_{0}=98$ for $V=8 \mu \mathrm{m} / \mathrm{s}, 140$ for $V=2$ and $4 \mu \mathrm{m} / \mathrm{s}$, and 198 for $V=1 \mu \mathrm{m} / \mathrm{s}$, which respectively correspond to a grid spacing $\Delta x[\mu \mathrm{m}]=1.75,2.50$, and 3.54 , and to an explicit time step $\Delta t[\mathrm{~s}] \simeq 0.0015,0.0035$, and 0.0069. As mentioned in Appendix $\mathrm{C}$, we used $\Delta x_{T}=24 \Delta x$ for PF simulations with a 1D temperature field, and $\Delta x_{T}=33 \Delta x$ when using a 3D 
temperature field. Using both 1D and 3D thermal fields, we assessed the effect of the thermal grid spacing on the microstructure for spatially extended simulations at $V=4 \mu \mathrm{m} / \mathrm{s}$ and found no significant difference when decreasing $\Delta x_{T}$ by up to a factor 3. For thermal fluctuations, we introduced noise onto the $\psi$ field with a strength $F=0.02[20,21,22,54]$. We imposed periodic boundary conditions in the $x$ and $y$ directions and symmetric boundaries along the $z$ boundaries. Most simulations were performed for a large system size, $L_{x} \times L_{y} \times L_{z}\left[\mu \mathrm{m}^{3}\right] \simeq 700 \times 700 \times 3444$ for $V=1 \mu \mathrm{m} / \mathrm{s}, 495 \times 495 \times 1495$ for $V=2 \mu \mathrm{m} / \mathrm{s}, 495 \times 495 \times 1115$ for $V=4 \mu \mathrm{m} / \mathrm{s}$, and $501 \times 501 \times 697$ for $V=8 \mu \mathrm{m} / \mathrm{s}$. For 3D TFC simulations, we used the same material and numerical parameters, and similar domain sizes except for $V=8 \mu \mathrm{m} / \mathrm{s}$ with $L_{x} \times L_{y} \times L_{z}\left[\mu \mathrm{m}^{3}\right] \simeq$ $578 \times 578 \times 697$.

We also performed simulations to identify stable spacing ranges of a hexagonal array by using a similar method as in Refs. [20, 21], i.e. progressively expanding the size of simulations of one quarter of cell growing at the stationary state to test the spacing upper limit of stability with respect to sidebranching. Simulations with three halves of cells in a hexagonal array were also performed to estimate the spacing lower limit of stability with respect to elimination $[20,21]$. In order to keep the same grid spacing and numerical parameters for all simulations at given alloy and process parameters, this expansion (or reduction) of the domain size is done by restarting a simulation with additional (or fewer) grid points in $x$ and $y$, initializing the fields by bilinear interpolation of steady-state fields from a previous simulation.

\section{Results and discussions}

\subsection{Transient interface dynamics}

Similarly as in experiments, at the beginning of each simulation, the planar solid-liquid interface is located close to the liquidus temperature of the alloy within a temperature gradient $G$ (black dashed line in Fig. 1). When pulling of the sample at a velocity $V$ is initiated, the interface recoils within the temperature frame, and on the liquid side of the interface, the solute concentration $c_{l}$ increases as a solute layer builds up ahead of the interface. The planar front then undergoes the Mullins-Sekerka instability and breaks down to small cells [38]. The onset of planar instability can be estimated using the constitutional undercooling criterion [39], i.e. it occurs when the solute concentration gradient in front of the interface - linked to the alloy concentration $c_{0}$, the interface solute partition coefficient $k$, the liquid solute diffusivity $D$, and the interface velocity $V_{i}$ - exceeds a critical value — related to $G$ and the alloy liquidus slope $m$ (cf. Eq. (5) discussed later). The resulting cells then compete with one another until they reach a stable spacing.

Experimental measurements of the time evolution of the interface position $z_{i}$ starting from rest $\left(z_{i}=0\right)$ are shown for different $V$ in Fig. 4a-d (black squares), and compared to results with three different thermal descriptions in PF simulations, namely FTA from Eq. (1) (blue thin solid lines), TDA from Eq. (2) (gray dotted lines), and 1D TFC from Eq. (3) (red thick solid lines). In all cases, the solid-liquid interface initially 
moves towards colder temperature $\left(z_{i}<0\right)$ because the pulling velocity $V$ is faster than the interface growth velocity $V_{i}$. After the destabilization of the planar interface, small cells move very fast towards the hotter temperature. Accordingly, a local maximum of $\left|z_{i}(L)\right|$ appears in Fig. 4 for both experiments and simulations. Then, primary cells approach a stationary temperature.

In the simulations using the FTA, the initial interface growth is fastest and overestimated compared to experiments. The planar front thus breaks down much earlier than in the experiments, with the red shaded areas in Fig. 4a-d showing the time range for planar destabilization observed in the experiments. Ultimately, cell tips stabilize relatively close to the liquidus temperature.

On the other hand, PF simulations using the TDA [30] or the newly introduced TFC yield better agreements with experiments. Compared to the FTA, not only is the time evolution of the interface position better estimated, but the time for the onset of morphological instability also occurs closer to the experimental observations. As seen in Fig. 4b, the coupled TFC provides a better estimation than the phenomenological TDA, while involving no adjustable parameter.

Despite the substantial improvement, the onset of morphological instability and the interface positions in both TDA and TFC still show some amount of deviation from experimental measurements at $V=1 \mu \mathrm{m} / \mathrm{s}$ (Fig. 4a). We attribute this discrepancy to possible residual convection effects, as discussed in Ref. [36]. These effects are expected to be more significant at lower velocities [29]. In the following, we focus on simulations at $V \geq 2 \mu \mathrm{m} / \mathrm{s}$.

The bottom plot in Fig. 1 illustrates the difference in temperature fields at the stationary state when $V=4 \mu \mathrm{m} / \mathrm{s}$ for the FTA, TDA and TFC. In the initial state with $G=19 \mathrm{~K} / \mathrm{cm}$, all are equivalent to the FTA, and the interface is located at $z_{i}=0$. In the stationary state, cell tip temperatures (hollow circles) differ only slightly, but the final interface position is much further from the initial position with the TFC or the TDA than with the FTA.

Furthermore, we illustrate the importance of the latent heat rejection by plotting in Fig. 4b the interface position evolution for a simulation at $V=2 \mu \mathrm{m} / \mathrm{s}$ without latent heat contribution, i.e. neglecting the last term in Eq. (3). The corresponding TFC simulation (red dashed line) severely underestimates the total recoil at the stationary state, which is consistent with discussions of the TDA in Ref. [30]. In addition, the lack of latent heat leads to the earlier planar destabilization, which could affect the primary spacing selection.

\subsection{Onset of morphological instability}

We illustrate the morphological evolution of the interface using the TFC simulation at $V=4 \mu \mathrm{m} / \mathrm{s}$ in Fig. 5. Snapshots correspond to plus symbols marked on the $z_{i}(L)$ curve in Fig. 4c (as well as in the central column of Fig. 6). When the planar interface becomes morphologically unstable (Fig. 5a), while the structure is not cellular yet, a first length scale emerges between individual bumps on the interface. As 
tips accelerate, they grow into small cells (Fig. 5b). These cells then progressively grow and compete with neighbor cells (Fig. 5c-e). After successive hierarchical elimination events, a primary spacing is selected (Fig. 5e), which exhibits a limited evolution after $L=2.97 \mathrm{~mm}$.

In Fig. 6, we show the simulated evolution of the liquid interface concentration $c_{l}$ (a - top row), the interface velocity $V_{i}$ (b - center row), and the overall selected spacing $\Lambda$ (c - bottom row) for different pulling velocities $V=2$ (left column), 4 (center column), and $8 \mu \mathrm{m} / \mathrm{s}$ (right column) with $G=19 \mathrm{~K} / \mathrm{cm}$, comparing results for the classical FTA (blue thin line) and the TFC (red thick line). The solute concentration at the interface on the liquid side $c_{l}$ builds up right after the sample is pulled in all simulations (Fig. 6a). Using the FTA (blue thin solid line), $c_{l}$ increases relatively fast; the planar instability occurs as $c_{l}$ is maximal; the interface then accelerates (Fig. 6b) as its solute concentration decreases; and the interface ultimately reaches steady position, velocity, and concentration. Comparatively, the concentration peak using the TFC (red thick solid line) is higher, but it takes a longer time to build up. The time for planar destabilization is hence in better agreement with experiments (red shaded area). The resulting peak velocity, associated to the growth of microstructure, is also lower using the TFC.

It is interesting to note that the differences of the peak velocity between the FTA and TFC simulations are amplified when increasing the pulling velocity (Fig. 6b). For example, the attenuation of the velocity peak is stronger at $V=8 \mu \mathrm{m} / \mathrm{s}$ compared to $2 \mu \mathrm{m} / \mathrm{s}$. When $V \geq 2 \mu \mathrm{m} / \mathrm{s}$ (Fig. $6 \mathrm{~b}$ ), the peak velocity with the FTA calculation tends to occur earlier in the growth length $L=V t$ at a lower pulling velocity, however this tendency in the TFC is inverse. Consequently, the difference of onset of cell growth between the two types of simulations becomes larger at a higher $V$.

The steady-state primary spacings are larger using the FTC simulation (Fig. 6c) and once more, the difference between the two simulations increases with pulling velocity. The larger primary spacings obtained in TFC simulations could result from the lower velocity peaks. However, those results evidence the major influence of limited heat diffusion and latent heat release on the interface dynamics, and as a result on the primary spacing selection, especially for large pulling velocities. Comparing the initial transient of primary spacing (Fig. 6c), we can see that the TFC simulation is in reasonable quantitative agreement with experiments; however, a discrepancy remains for $V=8 \mu \mathrm{m} / \mathrm{s}$ which could be linked to the large experimental overshoot that was not reproduced in a numerical simulation.

In all cases, the interface velocity $V_{i}=V+\mathrm{d} z_{i} / \mathrm{d} t$ slowly increases soon after the sample moves. Classical theory stipulates that a planar interface can destabilize when $V_{i}$ is larger than a critical velocity $V_{c}$, which can be estimated using the constitutional undercooling criterion [38, 39]

$$
V_{c}=\frac{G D}{|m| \Delta c_{i}},
$$

where $m$ is the liquidus slope ( $m=-1.365 \mathrm{~K} / \mathrm{wt} \%$ for our considered SCN alloy), and $\Delta c_{i}$ is the concentration gap across the interface. Considering a constant temperature gradient $G$ and $\Delta c_{i}=c_{0}(1 / k-1)$ at the 
alloy solidus temperature (i.e. the temperature of a stationary stable planar interface), the critical velocity is $V_{c}^{0}=0.118 \mu \mathrm{m} / \mathrm{s}$ for our current control parameters. Fig. $6 \mathrm{~b}$ shows that this velocity, shown as a black dashed line, is reached very early by $V_{i}$ in all simulations.

We modify the calculation of $V_{c}$ by accounting for the time evolution of $\Delta c_{i}(t)=(1-k) c_{l}(t)$. In order to discard effects of the planar destabilization on $\Delta c_{i}(t)$, we use $c_{l}(t)$ from both FTA and TFC simulations using a reduced size in the $x$ and $y$ directions such that the interface remains planar similarly as in $1 \mathrm{D}$ simulations (instead of using the concentrations directly from Fig. 6a). The resulting calculated $V_{c}(t)$, in color dashed lines in Fig. 6b, which tend to $V_{c}^{0}$ as the planar interface approaches the solidus temperature, give an estimation of the instantaneous critical velocity in both FTA (blue thin) and TFC (red thick) simulations. Since the solute build up is slower considering the TFC (Fig. 6a), its $V_{c}(t)$ is relatively higher than with the FTA. Hence, the combined facts that $V_{i}(t)$ is lower and that $V_{c}(t)$ is higher using the TFC explain why the planar destabilization occurs at a significantly later time.

However, in all cases, the instability becomes noticeable significantly later than the time $t=t_{0}$ at which $V_{i}(t)$ intersects $V_{c}(t)$ in Fig. 6b. This delay is linked to the time it takes for the initial wavelength to be amplified, and is thus expected to depend on the amplitude of thermal fluctuations [23].

Fig. 7a shows initial average spacings measured at the onset of instability for different pulling velocities. This initial spacing $\Lambda_{0}$ decreases as the pulling velocity increases in both experiments (black squares), FTA (blue diamonds), and TFC simulations (red circles). Error bars in Fig. 7a correspond to minimum and maximum spacings measured in experiments.

Linear stability analysis $[38,23,56]$ can be used to estimate the critical initial spacing $\Lambda_{c}$ at the onset of planar destabilization using the dispersion relation giving the exponential amplification rate $\omega$ of small amplitude interface perturbations of wavenumber $k_{n}$. This relation is given by Eq. (32) in Ref. [56], which is expressed here by scaling length with $l_{d}=D / V$ and time with $D / V^{2}$

$$
\begin{array}{r}
\omega\left[1-\frac{1-k}{r} \xi\right]-k(1+\dot{\xi}) g_{c}(t)-\frac{\dot{\xi}}{r}= \\
{[q+(1+\dot{\xi})(k-1)]\left[-g_{c}(t)-\frac{1}{r}-\frac{d_{0}}{l_{d}} k_{n}^{2}\right] .}
\end{array}
$$

where

$$
q=\frac{1+\dot{\xi}}{2}+\left[\frac{(1+\dot{\xi})^{2}}{4}+k_{n}^{2}\right]^{1 / 2}
$$

in the quasi-stationary limit where $\omega \ll k_{n}$. Here, we adapt the ratio $r$ between the thermal length $l_{T}$ and diffusion length in order to account for an instantaneous thermal length $l_{T}(t)=|m| \Delta c_{i}(t) / G$, which yields $r(t)=l_{T}(t) / l_{d}=V / V_{c}(t)$. In order to evaluate an instantaneous interface position $\xi=\xi(t)$ between the liquidus and solidus temperatures, and its time derivative $\dot{\xi}=\mathrm{d} \xi / \mathrm{d} t$, we use the relations [56] for the local 
equilibrium condition at the interface

$$
\frac{c_{l}-c_{0}}{c_{0}(1 / k-1)}=1-\frac{\xi}{r},
$$

and the interface velocity

$$
V_{i}=V(1+\dot{\xi})
$$

The solute gradient at the interface on the liquid side $g_{c}(t)$ is given by

$$
g_{c}(t)=-(1+\dot{\xi})\left[1-\frac{\xi}{r}(1-k)\right] \text {. }
$$

The temperature gradient presumably remains the initial $G=19 \mathrm{~K} / \mathrm{cm}$ since $G$ at the onset of morphological instability is close to the initial $G$, for instance $G \approx 17.8 \mathrm{~K} / \mathrm{cm}$ in the TFC for $V=8 \mu \mathrm{m} / \mathrm{s}$. Then, using PF data for $c_{l}(t), V_{i}(t)$, and $V_{c}(t)$ from Fig. 6a-b between the time $t_{0}$ (up triangles in Fig. 7b) when $V_{i}(t)$ intersects $V_{c}$ and $t_{1}$ (down triangles in Fig. 7b) at the onset of morphological instability, we calculate $\omega\left(k_{n}\right)$ at a time $t$. From the results of $\omega\left(k_{n}\right)$ at different $t$, we find the critical time $t=t_{c}$ (filled circle dots in Fig. $7 \mathrm{~b})$, where the amplification rate becomes $\omega\left(k_{n}\right) \approx 0$ and $\mathrm{d} \omega\left(k_{n}\right) / \mathrm{d} k_{n} \approx 0$ at the critical wavenumber $k_{n}=k_{c}$. Then, we calculate the critical initial spacing at $k_{c}$,

$$
\Lambda_{c}=\frac{2 \pi l_{d}}{k_{c}} .
$$

In Fig. $7 \mathrm{a}$, the predicted $\Lambda_{c}$ from both FTA (blue thin line) and TFC (red thick line) are close to the experimental measurements.

Fig. 7b shows $t_{0}, t_{c}$, and $t_{1}$ measured in PF simulations using the FTA (blue symbols) and the TFC (red symbols), which decrease as $V$ increases. As already observed earlier, the TFC improves the agreement of predicted onset time for the morphological instability with respect to experimental measurements (black vertical error bars, equivalent to red shaded areas in Fig. 4 and Fig. 6).

One interesting observation is that the time interval between $t_{0}$ and $t_{1}$ in both FTA (blue plus) and TFC (red cross) decreases as a power law of $V$, namely with $t_{1}-t_{0} \sim V^{-\alpha}$, as shown in the subset of Fig. 7b using a log-log scale. The power law exponent is $\alpha \approx 1.5$ for the FTA (blue thin line) and 0.9 for the TFC (red thick line). This indicates that the finite thermal diffusion and the latent heat release further delay the amplification of the interface perturbations. However, whereas all simulations here were performed using a noise strength $F=0.02$, a stronger noise amplitude could possibly affect the instability and yield earlier $t_{1}$.

\subsection{Stationary spacing selection}

As mentioned in the introduction, previous PF simulations of DECLIC-DSI experiments systematically predicted primary spacings smaller than those measured in the experiments [20,21, 22]. These simulations were at the time carried out using the FTA and the initially assessed partition coefficient $k=0.21[20,21]$. An accurate reevaluation of the partition coefficient for this specific alloy concentration through carefully 
controlled experiments lead to the current value $k=0.07$ [31]. Using this value of $k$, we estimated the stable spacing range and dynamically selected stationary spacing in spatially extended simulations for different $V$.

Fig. $8 \mathrm{a}$ shows the predicted steady state tip undercooling and the corresponding spacing ranges for $G=19 \mathrm{~K} / \mathrm{cm}$ and $V[\mu \mathrm{m} / \mathrm{s}]=1,2,4$, and 8 , from PF simulations using $k=0.07$ with the FTA and a similar procedure as in Refs. $[21,22,26]$. Corresponding steady tip radii appear in Fig. 8b. We find that 1D TFC simulations yield stable spacing ranges that tend to be wider than using the FTA, but only slightly, i.e. up to $\sim 10 \%$ lower (higher) than the minimum (maximum) spacing limit in Fig. 8a. Thus, Fig. 8 a shows stationary tip undercooling of a quarter of a cell in a hexagonal array as a function of the primary spacing [21], only for the FTA.

As shown in Fig. 8b, all experimentally measured average spacings (black squares), which closely follow a $\Lambda \sim V^{-1 / 4}$ scaling law [11, 16, 8, 7], as well as their standard deviations throughout the sample (error bars), fall within the calculated stable spacing ranges (boxes). Yet, despite fairly similar stability ranges using FTA or TFC with a 1D temperature field (Eq. 3), dynamically selected spacings in spatially extended simulations using the TFC (red hollow circles) are systematically larger than with the FTA (blue diamonds). Hence, the more accurate TFC improves the agreement between predicted and measured spacings. The higher selected spacing using the TFC could be linked to slower solute build ahead of the interface and to the resulting lower velocity peak after planar destabilization, as exemplified in Fig. 6a-b. As observed in Fig. 7a, the initially selected spacing, also fairly similar using FTA and TFC, may not be influential on the final microstructure selection.

In order to assess the effect of a 3D temperature field, we also performed spatially extended simulations for $V[\mu \mathrm{m} / \mathrm{s}]=1,2,4$, and 8 . As shown in Fig. 8c, with a 3D TFC in (red cross symbols), the resulting lateral heat transport results in larger spacings, hence in even better agreement with experimental measurements. While the stable spacing ranges themselves remain weakly affected by the representation of the $1 \mathrm{D}$ thermal field, the dynamical selection of a higher spacing within a stable range when using a TFC stems from: (i) the lower velocity peak upon planar destabilization that contributes in selecting a higher initial spacing (Fig. 6b), and (ii) a locally lower temperature gradient when using a TFC compared to FTA or TDA thermal representations (Fig. 1). Both effects also explain why these effects increase with increasing velocity V (Fig. 8c).

While we did not calculate the entire stable ranges for all velocities using a three-dimensional thermal field, we found that the minimum spacing limit $\Lambda_{\min }$ can increase due to lateral heat transport. For $V=$ $2 \mu \mathrm{m} / \mathrm{s}$, we assessed that $\Lambda_{\min }$ with a $3 \mathrm{D}$ TFC is about $50 \mu \mathrm{m}$ larger, i.e. about $50 \%$ higher, than using a FTA or 1D TFC. Thus, the larger selected spacing is likely also linked to a larger minimum stable spacing $\Lambda_{\min }$ when accounting for lateral heat diffusion. The physical origin of this increase of $\Lambda_{\min }$ is discussed further below.

The lateral temperature distribution along $x$ and $y$ directions in the spatially extended 3D TFC simula- 
tions is illustrated for $V=4 \mu \mathrm{m} / \mathrm{s}$ in Fig. 9. As expected, the most advanced primary tips correspond to hotter zones ( $T_{\text {Max }}$, red contour lines). The most important observation from Fig. 9 is that temperature deviations from a purely 1D temperature field are of the order of $10^{-3} \mathrm{~K}$. Indeed, in Fig. 9, iso-values of the temperature field within the $z=z_{i}$ plane are shown with steps of $0.5 \times 10^{-3} \mathrm{~K}$ from $T_{\text {Max }}$ in all panels. Shortly after planar destabilization at $t=494 \mathrm{~s}$, near the peak interface velocity (cf. Fig. 6b), Fig. 9a shows that this temperature difference is about $5 \times 10^{-3} \mathrm{~K}$. This is the largest temperature difference observed in the $z=z_{i}$ plane, since at later times $t>500 \mathrm{~s}$ (Fig. $9 \mathrm{~b}-\mathrm{d}$ ), it does not exceed $2 \times 10^{-3} \mathrm{~K}$.

Such a small temperature difference of about $5 \times 10^{-3} \mathrm{~K}$ corresponds to a difference in the longitudinal position of the tip of about $2.63 \mu \mathrm{m}$ considering the nominal temperature gradient $\mathrm{G}=19 \mathrm{~K} / \mathrm{cm}$. While this height difference appears negligible when compared to the total length of the adiabatic zone $(2.34 \mathrm{~cm})$, it is less so when compared to other crucial length scales involved in the spacing selection, namely the diffusion length $\mathrm{D} / \mathrm{V}$, ranging from 34 to $270 \mu \mathrm{m}$, and the cell/dendrite tip radius, plotted in Fig. 8b and ranging from about 15 to $75 \mu \mathrm{m}$ in the steady state. Both lengths are even locally much smaller during the planar destabilization and sudden acceleration stage critical to the initial spacing selection. Hence, although $5 \times 10^{-3} \mathrm{~K}$ is small compared to overall temperature differences within the entire experimental setup, this is sufficient to induce a difference in final average primary spacings of the order of tens of percent (Fig. 8c). This observation not only illustrates the widely multiscale aspect of microstructural selection in directional solidification of an alloy, but also further highlights why the effect of the thermal field is more pronounced at a higher pulling velocity (Fig. 8).

A second effect of the 3D thermal field calculation is the possibility to stabilize smaller cells that lag behind their neighbors but do not seem to be eliminated. Such a cell is illustrated in the top left part in Fig. 9d (circled with a green dotted line). In contrast, when using a 1D thermal field, if a cell starts recoiling within the temperature field, it usually leads to its elimination. This behavior stems from a counterintuitive effect of the lateral rejection of heat during directional solidification. Our interpretation is that the heat rejected on the side of the leading cells actually increases the temperature gradient directly ahead of the trailing cell, which then promotes its growth. The trailing cell thus finds itself in a stable state with competing effects of solute, promoting its elimination, and heat, promoting its growth. Hence, while it could have been expected to yield melting and subsequent elimination of trailing cells, the heat rejection by leading cells in fact has a stabilizing effect, which is reflected in a $50 \%$ larger minimum stable spacing $\Lambda_{\text {min }}$ in 3D TFC compared to 1D TFC or FTA.

Despite the significant improvement, $\Lambda$ remains larger in the experiments than in spatially extended PF simulations, which also do not exhibit the small $\Lambda(t)$ overshoot before slowly decreasing to a stationary $\Lambda$ (see Figs. 3a and 6c). In detailed observations of the first stages of pattern formation during experiments, we find that grains misoriented with respect to the temperature gradient lead to grain boundaries, which may play an important role in the evolution of primary spacing. The remaining discrepancy could be linked to 
additional uncertainties on exact alloy or processing parameters, to an underestimation of thermal diffusivity inside the crucible, or to a size effect in the PF simulations containing a small number of cells (as low as only nine cells in spatially extended TFC simulations at $V=2 \mu \mathrm{m} / \mathrm{s}$ ). Furthermore, this latter quantization effect may lead to different selected final $\Lambda$ between two simulations using a different random number seed. (Only the result of one simulation per velocity is shown in Fig. 8.)

\subsection{Stationary tip undercooling}

Finally, we calculated the tip undercooling $\Delta$ in the FTC simulations in order to compare it to previously proposed analytical estimations [57, 58, 59]. Bower, Brody, and Flemings [57] proposed a tip undercooling given by

$$
\Delta_{\mathrm{BBF}}=\frac{D}{V l_{T}}
$$

Later, Karma and Pelcé [58, 59] proposed

$$
\Delta_{\mathrm{KP}}=\frac{f_{s} k+\left(1-f_{s}\right) \frac{D}{V l_{T}}}{1-f_{s}(1-k)},
$$

where $f_{s}$ is the solid fraction in a region behind the tip. We used the expression for a Saffman-Taylor finger [60] to calculate $f_{s}$. For the description for the Saffman-Taylor finger [61,62, 63], the finger width approaches $R=1 / 2$ of the channel width as surface tension anisotropy decreases. We used this finger width to estimate the fraction, yielding $f_{s}=R^{2}=1 / 4$ in $3 \mathrm{D}$. As shown in Table 2 , the predicted $\Delta_{\mathrm{KP}}$ with $f_{s}=0.25$ shows better agreements with the TFC results than $\Delta_{\mathrm{BBF}}$, which is consistent with previous single cell simulation results [53]. It is worth noting that, $f_{s}$ determined in the directional solidification experiments is usually larger than 0.25 from the Saffman-Taylor finger [30], which leads to larger $\Delta_{\mathrm{KP}}$.

\section{Summary and outlook}

We studied microstructure dynamics in microgravity experiments performed in the DECLIC Directional Solidification Insert aboard the ISS. We used three-dimensional quantitative phase-field (PF) simulations to discuss the influence of the thermal field, and its representation in the model, upon transient solid-liquid interface dynamics, and the selection of spacings in resulting microstructures.

As experimentally shown in Ref. [30], combined effects of latent heat release and finite heat diffusion in the sample lead to discrepancies in the interface evolution compared to classical approaches that assume a frozen temperature field, such as in the classical model by Warren and Langer (WL) [23]. However, the WL model can be phenomenologically modified to account for a time-dependent thermal drift of the system, so as to better describe the experimentally measured initial recoil of the planar interface within the temperature field [30]. 
In the current article, we studied different representations of the thermal field in the DECLIC-DSI setup within quantitative PF simulations. Namely, we compared three representations: (i) the classical Frozen Temperature Approximation (FTA); (ii) the phenomenological Thermal Drift Approximation (TDA) [30]; and (iii) a one-dimensional Thermal Field Calculation (TFC) coupled with time-dependent 3D PF simulations.

Simulation results show a much better agreement with measured transient interface recoil using the TDA or TFC than with the classical FTA (Fig. 4). The idealized FTA yields a stationary interface position relatively too close to the initial position (i.e. the liquidus temperature location), which is corrected with more accurate TDA and TFC. In addition, the simulations with the TDA and TFC also predict a time for the onset of morphological instability in much better agreement with experimental observations (Fig. 4).

During the planar interface recoil within the temperature field, the TFC leads to a slower increase of the interface solute concentration $c_{l}$ than with the classical FTA (Fig. 6a). The resulting interface velocity in the former thus increases relatively slower. Both effects contribute to the later occurrence of the planar morphological instability, which we have predicted by comparing the interface velocity $V_{i}(t)$ to an instantaneous constitutional undercooling critical velocity $V_{c}(t)$ that takes into account the time-dependent interface concentration (Fig. 6b). The instability condition $V_{i}(t)>V_{c}(t)$ is reached much later in the TFC simulations than using the FTA. However, noticeable acceleration of the interface occurs later than the time to reach $V_{i}(t)>V_{c}(t)$, due to the time necessary to amplify the initial perturbation of the interface.

The different interface acceleration profiles after morphological instability also lead to different selected microstructural lengths. While the stable spacing range for stationary state growth is similar for the FTA and TFC due to the interface position and local temperature gradient being almost identical (Fig. 1), dynamically selected spacings are higher with the TFC. Our interpretation is that it is linked to the slower acceleration and the lower peak velocity after planar destabilization (Fig. 6b).

We find that initially measured spacings $\Lambda_{0}$ in the experiments and the simulations can be also predicted by using an analytical stability analysis as proposed in Ref. [56]. Using the time-dependent $c_{l}(t), V_{i}(t)$, and $V_{c}(t)$ (Fig. 6a-b) from PF simulations at a critical time $t_{c}$, we calculate a critical wavelength and the resulting critical spacing $\Lambda_{c}$ (Fig. 7a). The resulting predictions show good agreements with the measurements. In addition, these similar initial spacings in the experiments and simulations indicate that the difference in selected steady-state spacing could mostly come from the history of $c_{l}$ and $V_{i}$ (Fig. 6b) rather than the initial array spacings.

Simulations do not predict the spacing $\Lambda(t)$ overshoot and slow decrease observed in experiments (Fig. 6c), and still tend to underestimate stationary selected spacing (Fig. 8b). However, the introduction of the TFC in PF simulations substantially improves the agreement between predicted and experimentally measured spacings. Specifically, the selected spacings in the 3D TFC simulations are closer to the experimental measurements (Fig. 8b). Thus, the thermal diffusion within the crucible after the morphological instability also plays an important role in spacing selection. 
Possible additional reasons for the global spacing underestimation in simulations include the influence of the crystal misorientation with respect to the temperature gradient [22]. Moreover, the thermal diffusivity of the SCN alloy, which is lower than that of the wall or the considered effective $D_{T}$, could be at the origin of the initial spacing overshoot in the experiments. Detailed studies are currently underway in these directions.

In conclusion, current results highlight the effect of subtle differences in the thermal field upon microstructure selection, and the importance of its accurate description in models. Moreover, they emphasize the importance of the history of concentration at the interface and the interface velocity on primary spacing selection mechanisms.

\section{Acknowlegements}

This study was supported by the National Aeronautics and Space Administration NASA Grants No. NNX12AK54G and NNX16AB54G, and the French Space Agency CNES (Microstructures de solidification 3D - MISOL3D project). We would like to thank J.-M. Debierre (Aix-Marseille Université) for valuable discussions.

\section{Appendix A. Frozen Temperature Approximation}

Following the detailed derivation in Ref. [48], applying the FTA, i.e. Eq. (1), and making the nonlinear change of variable $\varphi \equiv \tanh (\psi / \sqrt{2})[49,51]$, the evolution of the preconditioned phase field $\psi$ and of the normalized solute concentration $U$ with time $t$ in a lab frame obeys

$$
\begin{array}{r}
F_{T 1}(z, t) a_{s}(\mathbf{n})^{2} \frac{\partial \psi}{\partial t}=\vec{\nabla}\left[a_{s}(\mathbf{n})^{2}\right] \vec{\nabla} \psi \\
+a_{s}(\mathbf{n})^{2}\left[\nabla^{2} \psi-\varphi \sqrt{2}|\vec{\nabla} \psi|^{2}\right] \\
+\sum_{m=x, y, z}\left[\partial_{m}\left(|\vec{\nabla} \psi|^{2} a_{s}(\mathbf{n}) \frac{\partial a_{s}(\mathbf{n})}{\partial\left(\partial_{m} \psi\right)}\right)\right] \\
+\sqrt{2}\left[\varphi-\lambda_{c}\left(1-\varphi^{2}\right) F_{T 2}(z, t)\right], \\
(1+k-(1-k) \varphi) \frac{\partial U}{\partial t}=\widetilde{D} \vec{\nabla} \cdot[(1-\varphi) \vec{\nabla} U] \\
+\vec{\nabla} \cdot\left[(1+(1-k) U) \frac{\left(1-\varphi^{2}\right)}{2} \frac{\partial \psi}{\partial t} \frac{\vec{\nabla} \psi}{|\vec{\nabla} \psi|}\right] \\
+[1+(1-k) U] \frac{\left(1-\varphi^{2}\right)}{\sqrt{2}} \frac{\partial \psi}{\partial t},
\end{array}
$$

with

$$
U \equiv \frac{1}{1-k}\left[\frac{2 c / c_{l}^{0}}{(1+k)-(1-k) \varphi}-1\right]
$$


where $c_{l}^{0}=c_{\infty} / k$ is the equilibrium concentration of the liquid at the reference temperature $T_{0}$ (here the alloy solidus) with $c_{\infty}$ the nominal composition, and $k$ is the partition coefficient $(0<k<1)$. For a cubic symmetry material, the standard anisotropy function is

$$
a_{s}(\mathbf{n})=\left(1-3 \varepsilon_{4}\right)\left[1+\frac{4 \varepsilon_{4}}{1-3 \varepsilon_{4}}\left(n_{x}^{4}+n_{y}^{4}+n_{z}^{4}\right)\right],
$$

where $\varepsilon_{4}$ is the anisotropy strength [46] and $\left(n_{x}, n_{y}, n_{z}\right)$ are the components of the interface normal vector n. The coupling factor is given by

$$
\lambda_{c}=a_{1} \frac{W}{d_{0}} .
$$

Space and time are scaled with respect to the diffuse interface width $W$ and a relaxation time $\tau_{0}$, respectively, and thus the dimensionless diffusivity $\widetilde{D}$ is

$$
\widetilde{D}=\frac{D \tau_{0}}{W^{2}}=a_{1} a_{2} \frac{W}{d_{0}},
$$

with the constants $a_{1}=5 \sqrt{2} / 8$ and $a_{2}=47 / 75[45,46]$. The capillarity length

$$
d_{0}=\frac{\Gamma}{|m| c_{\infty}(1 / k-1)}
$$

with $\Gamma$ the Gibbs-Thomson coefficient of the solid-liquid interface, and $m$ the alloy liquidus slope $(m<0)$. The two thermal terms in Eq. (A.1) are

$$
\begin{aligned}
& F_{T 1}(z, t)=\left[1-(1-k) \frac{z}{\tilde{l}_{T}}\right], \\
& F_{T 2}(z, t)=\left[U+\frac{z}{\tilde{l}_{T}}\right],
\end{aligned}
$$

where the dimensionless thermal length $\widetilde{l}_{T}$ is

$$
\tilde{l}_{T}=\frac{l_{T}}{W}=\frac{l_{T}}{d_{0}} \frac{1}{W / d_{0}} .
$$

Similarly as in previous studies [48, 51, 52, 26, 53], we solve Eqs. (A.1)-(A.2) on a regular cubic finite difference grid of element size $\Delta x$, using a explicit Euler time stepping scheme with a constant time step

$$
\Delta t=R_{S} \frac{(\Delta x)^{2}}{6 D}
$$

where $0<R_{S}<1$ is a constant, here chosen as $R_{S}=0.9$.

\section{Appendix B. Thermal Drift Approximation}

Replacing the FTA, Eq. (1), by the TDA, Eq. (2), the derivation of the equation for the evolution of $\psi$ (e.g. starting from Eq. (58) in Ref. [48]) yields the thermal functions

$$
\begin{aligned}
& F_{T 1}(z, t)=\left[1-(1-k) \frac{z+\Delta \tilde{z}_{T}\left(1-e^{-t / \tilde{\tau}_{d}}\right)}{\widetilde{l}_{T}}\right], \\
& F_{T 2}(z, t)=\left[U+\frac{z+\Delta \tilde{z}_{T}\left(1-e^{-t / \tilde{\tau}_{d}}\right)}{\widetilde{l}_{T}}\right],
\end{aligned}
$$


where the terms for the thermal drift (i.e. the total shift $\Delta z_{T}$ and the delay time $\tau_{d}$ ) are scaled as

$$
\begin{aligned}
\Delta \tilde{z}_{T} & =\frac{\Delta z_{T}}{W}, \\
\tilde{\tau}_{d} & =\frac{\tau_{d}}{\tau_{0}} .
\end{aligned}
$$

Hence, the evolution of the $\psi$ field follows Eq. (A.1) using Eqs. (B.1) and (B.2). This description does not affect the evolution of the $U$ field, which still evolves with Eq. (A.2).

\section{Appendix C. Thermal Field Calculation}

Considering a one-dimensional temperature field $T(z, t)$, the thermal functions in Eq. (A.1) can be written as

$$
\begin{aligned}
& F_{T 1}(z, t)=\left[1-(1-k) \frac{T(z, t)-T_{0}}{\Delta T_{0}}\right], \\
& F_{T 2}(z, t)=\left[U+\frac{T(z, t)-T_{0}}{\Delta T_{0}}\right]
\end{aligned}
$$

where $\Delta T_{0}=|m| c_{\infty}(1 / k-1)$ is the alloy freezing range, and $T_{0}$ is the reference (solidus) temperature. The $U$ field still evolves with time following Eq. (A.2).

The evolution of the temperature field with Eq. (3) is also solved explicitly using a same time step $\Delta t$ as for Eqs. (A.1)-(A.2), but on a separate finite difference grid of step size $\Delta x_{T}$. Using a similar stability condition for the $1 \mathrm{D}$ as Eq. (A.11)

$$
\Delta t=R_{T} \frac{\left(\Delta x_{T}\right)^{2}}{2 D_{T}}
$$

where the prefactor is here set to $R_{T}=0.6$. This yields a relation between the two grid steps

$$
\Delta x_{T}=\sqrt{\frac{R_{S}}{R_{T}} \frac{D_{T}}{3 D}} \Delta x
$$

Since the thermal diffusivity $D_{T}$ is much higher than the solute diffusivity $D, \Delta x_{T}$ is larger than $\Delta x$. Hence, Eq. (3) is solved as

$$
\begin{aligned}
\partial_{t} T & =V \partial_{z} T+D_{T} \partial_{z z} T \\
& +\frac{\Delta h_{f}}{c_{p}} \sum_{z}^{z+\Delta x_{T}} \frac{\sum_{x, y} \dot{\varphi}(x, y, z) / 2}{\left(N_{x} \times N_{y}\right)} \frac{\Delta x}{\Delta x_{T}},
\end{aligned}
$$

i.e. with the latent heat term integrated over all grid points along $x$ and $y$ dimensions, respectively with $N_{x}$ and $N_{y}$ grid points in the domain within a range $\left[z, z+\Delta x_{T}\right]$. We used $\Delta x_{T}=24 \Delta x$ for PF simulations with a $1 \mathrm{D}$ temperature field. 
For the 3D temperature field, we solve Eq. (4) as

$$
\begin{aligned}
\partial_{t} T & =V \partial_{z} T+D_{T}\left(\partial_{x x}+\partial_{y y}+\partial_{z z}\right) T \\
& +\frac{\Delta h_{f}}{c_{p}} \sum_{z}^{z+\Delta x_{T}} \sum_{y}^{y+\Delta x_{T}} \sum_{x}^{x+\Delta x_{T}} \frac{\dot{\varphi}(x, y, z)}{2}\left(\frac{\Delta x}{\Delta x_{T}}\right)^{3}
\end{aligned}
$$

at the same time step $\Delta t$ for the other fields and $\Delta x_{T}=33 \Delta x$ which satisfy the stability condition for a 3D temperature field. Therefore, the latent heat is integrated over the grid points within $\left[x, x+\Delta x_{T}\right]$, $\left[y, y+\Delta x_{T}\right]$, and $\left[z, z+\Delta x_{T}\right]$ along the $x, y$, and $z$ axes, respectively.

Dirichlet boundary conditions for the temperature field on $z$ boundaries are extracted from axisymmetric thermal simulations of the experimental setup using the CrysVUn software [30].

\section{References}

[1] M.C. Flemings, Solidification Processing, McGraw-Hill, New York, 1974.

[2] W. Kurz and D.J. Fisher, Fundamentals of Solidification, third ed., Trans Tech, Aedermannsdorf, 1992.

[3] J.A. Dantzig and M. Rappaz, Solidification, EPFL Press, Switzerland, 2009.

[4] P.W. Bridgman, Bridgman: Certain Physical Properties of Single Crystals of Tungsten, Antimony, Bismuth, Tellurium, Cadmium, Zinc, and Tin, Proc. Am. Acad. Arts Sci. 60 (1925) 305-383.

[5] R. Mehrabian, M. Keane, M.C. Flemings, Interdendritic fluid flow and macrosegregation; influence of gravity, Metall. Trans. 1 (1970) 1209-1220.

[6] H. Jamgotchian, N. Bergeon, D. Benielli, Ph. Voge, B. Billia, R. Guérin, Localized Microstructures Induced by Fluid Flow in Directional Solidification, Phys. Rev. Lett. 87 (2001) 166105.

[7] J.D. Hunt, Solidification and Casting of Metals, The Metals Society, London, 1979, pp. 3-9.

[8] W. Kurz, D.J. Fisher, Dendrite growth at the limit of stability: tip radius and spacing, Acta Metall. 29 (1981) $11-20$.

[9] K. Somboonsuk, J.T. Mason, R. Trivedi, Interdendritic Spacing: Part I. Experimental studies, Metall. Trans. A 15A (1984) 967-975.

[10] R. Trivedi, K. Somboonsuk, Constrained dendritic growth and spacing, Mater. Sci. Eng. 65 (1984) 65-74.

[11] R. Trivedi, Interdendritic Spacing: Part II. a comparison of theory and experiment, Metall. Trans. A 15A (1984) 977-982.

[12] K. Somboonsuk, R. Trivedi, Dynamical studies of dendritic growth, Acta Metall. 33 (1985) $1051-1060$.

[13] R. Trivedi, K. Somboonsuk, Pattern formation during the directional solidification of binary systems, Acta Metall. 33 (1985) 1061-1068.

[14] H. Esaka, W. Kurz, Columnar dendrite growth: experiments on tip growth, J. Cryst. Growth 72 (1985) 578-584.

[15] R.N. Grugel, Y. Zhou, Primary dendrite spacing and the effect of off-axis heat flow, Metall. Trans. A 20A (1989) 969-973.

[16] S.H. Han, R. Trivedi, Primary spacing selection in directionally solidified alloys, Acta Metall. Mater. A 42 (1994) 25-41.

[17] M.E. Glicksman, M. B. Koss, E. A. Winsa, Dendritic growth velocities in microgravity, Phys. Rev. Lett. 73 (1994) 573-576.

[18] S. Akamatsu, H. Nguyen-Thi, In situ observation of solidification patterns in diffusive conditions, Acta Mater. 108 (2016) $325-346$.

[19] N. Bergeon, A. Ramirez, L. Chen, B. Billia, J. Gu, R. Trivedi, Dynamics of interface pattern formation in 3D alloy solidification: first results from experiments in the DECLIC directional solidification insert on the International Space Station, J. Mater. Sci. 46 (2011) 6191-6202.

[20] N. Bergeon, D. Tourret, L. Chen, J.-M. Debierre, R. Guérin, A. Ramirez, B. Billia, A. Karma, R. Trivedi, Spatiotemporal dynamics of oscillatory cellular patterns in three-dimensional directional solidification, Phys. Rev. Lett. 110 (2013) 226102. 
[21] D. Tourret, J.-M. Debierre, Y. Song, F.L. Mota, N. Bergeon, R. Guérin, R. Trivedi, B. Billia, and A. Karma, Oscillatory cellular patterns in three-dimensional directional sodlification, Phys. Rev. E 92 (2015) 042401.

[22] J. Pereda, F.L. Mota, L. Chen, B. Billia, D. Tourret, Y. Song, J.-M. Debierre, R. Guérin, A. Karma, R. Trivedi, N. Bergeon, Experimental observation of oscillatory cellular patterns in three-dimensional solidification, Phys. Rev. E 95 (2017) 012803 .

[23] J.A. Warren, J.S. Langer, Prediction of dendritic spacings in a directional-solidification experiment, Phys. Rev. E 47 (1993) 2702-2712.

[24] S. Lu, J.D. Hunt, A numerical analysis of dendritic and cellular array growth: the spacing adjustment mechanisms, J. Cryst. Growth 123 (1992) 17-34.

[25] J.D. Hunt, S. Lu, Numerical modeling of cellular/dendritic array growth: spacing and structure predictions, Metallurgical and Materials Transactions A 27 (1996) 611-623.

[26] B. Echebarria, A. Karma, S. Gurevich, Onset of sidebranching in directional solidification, Phys. Rev. E 81 (2010) 021608.

[27] D. Tourret, A. Karma, Multiscale dendritic needle network model of alloy solidification, Acta Mater. 61 (2013) 6474-6491.

[28] D. Tourret, A.J. Clarke, S.D. Imhoff, P.J. Gibbs, J.W. Gibbs, A. Karma, Three-Dimensional multiscale modeling of dendritic spacing selection during Al-Si directional solidification, JOM 67 (2015) 1776-1785.

[29] A.J. Clarke, D. Tourret, Y. Song, S.D. Imhoff, P.J. Gibbs, J.W. Gibbs, K. Fezzaa, A. Karma, Microstructure selection in thin-sample directional solidification of an $\mathrm{Al}-\mathrm{Cu}$ alloy: in situ X-ray imaging and phase-field simulations, Acta Mater. 129 (2017) 203-216.

[30] F.L. Mota, N. Bergeon, D. Tourret, A. Karma, R. Trivedi, B. Billia, Initial transient behavior in directional solidification of a bulk transparent model alloy in a cylinder, Acta Mater. 85 (2015) 362-377.

[31] F.L. Mota, L.M. Fabietti, N. Bergeon, L.L. Strutzenberg, A. Karma, B. Billia, R. Trivedi, Quantitative determination of the solidus line in the dilute limit of succinonitrilecamphor alloys, J. Cryst. Growth 447 (2016) 31-35.

[32] N. Bergeon, R. Trivedi, B. Billia, B. Echebarria, A. Karma, S. Liu, C. Weiss, N. Mangelinck, Necessity of investigating microstructure formation during directional solidification of transparent alloys in 3D, Advances in Space Research 36 (2005) 80-85.

[33] R. Marcout, G. Raymond, B. Martin, G. Cambon, B. Zappoli, F. Duclos, S. Barde, D. Beysens, Y. Garrabos, C. Lecoutre, B. Billia, DECLIC: a facility to investigate fluids and transparent materials in microgravity conditions in ISS, IAC-06-A2 (2006) 5 .

[34] G. Pont, S. Barde, B. Zappoli, F. Duclos, Y. Garrabos, C. Lecoutre, D. Beysens, B. Billia, N. Bergeon, N. MangelinckNoël, R. Marcout, DECLIC: a facility to study crystallization and critical fluids, 60th International Astronautical Congress, Daejeon, Republic of Korea (2009).

[35] N. Bergeon, C. Weiss, N. Mangelinck-Noël, B. Billia, Interferometric method for the analysis of dendrite growth and shape in 3D extended patterns in transparent alloys, Transactions of the Indian Institute of Metals 62 (2009) 455-460.

[36] F.L. Mota, Y. Song, J. Pereda, B. Billia, D. Tourret, J.-M. Debierre, R. Trivedi, A. Karma, N. Bergeon, Convection effects during bulk transparent alloy solidification in DECLIC-DSI and phase-field simulations in diffusive conditions, JOM 69 (2017) 1280-1288.

[37] N. Bergeon, F. L. Mota, L. Chen, D. Tourret, J. M. Debierre, R. Guérin, A. Karma, B. Billia, R. Trivedi, Dynamical microstructure formation in 3D directional solidification of transparent model alloys: in situ characterization in DECLIC Directional Solidification Insert under diffusion transport in microgravity, IOP Conf. Ser.: Mater. Sci. Eng. 84 (2015) 012077 .

[38] W.W. Mullins, R.F. Sekerka, Stability of a planar interface during solidification of a dilute binary alloy, J. Appl. Phys. 35 (1964) 444-451.

[39] W.A. Tiller, K.A. Jackson, J.W. Rutter, B. Chalmers, The redistribution of solute atoms during the solidification of 
metals, Acta Metall. 1 (1953) 428-437.

[40] V. Seetharaman, M.A. Eshelman, R. Trivedi, Cellular spacings - II. Dynamical studies, Acta Metall. 36 (1988) $1175-1185$.

[41] L.-Q. Chen, Phase-field models for microstructure evolution, Annu. Rev. Mater. Res. 32 (2002) $113-140$.

[42] W.J. Boettinger, J.A. Warren, C. Beckermann, A. Karma, Phase-field simulation of solidification, Annu. Rev. Mater. Res. 32 (2002) 163-194.

[43] J.J. Hoyt, M. Asta, A. Karma, Atomic and continuum modeling of dendritic solidification, Mater. Sci. Eng. R 41 (2003) 121-163.

[44] I. Steinbach, Why solidification? Why phase-field?, JOM 65 (2013) 1096-1102.

[45] A. Karma, W.J. Rappel, Phase-field method for computationally efficient modeling of solidification with arbitrary interface kinetics, Phys. Rev. E 53 (1996) R3017-R3020.

[46] A. Karma, W.J. Rappel, Quantitative phase-field modeling of dendritic growth in two and three dimensions, Phys. Rev. E 57 (1998) 4323-4349.

[47] A. Karma, Phase-field formulation for quantitative modeling of alloy solidification, Phys. Rev. Lett. 87 (2001) 115701.

[48] B. Echebarria, R. Folch, A. Karma, M. Plapp, Quantitative phase-field model of alloy solidification, Phys. Rev. E 70 (2004) 061604.

[49] K. Glasner, Nonlinear preconditioning for diffuse interfaces, J. Comp. Phys. 174 (2001) 695-711.

[50] J. Nickolls, I. Buck, M. Garland, K. Skadron, Scalable Parallel Programming with CUDA, Queue 6 (2008) 40-53.

[51] D. Tourret, A. Karma, Growth competition of columnar dendritic grains: A phase-field study, Acta Mater. 82 (2015) 64-83.

[52] D. Tourret, Y. Song, A.J. Clarke, A. Karma, Grain growth competition during thin-sample directional solidification of dendritic microstructures: A phase-field study, Acta Mater. 122 (2017) 220-235.

[53] S. Gurevich, A. Karma, M. Plapp, R. Trivedi, Phase-field study of three-dimensional steady-state growth shapes in directional solidification, Phys. Rev. E 81 (2010) 011603.

[54] J. Ghmadh, J.-M. Debierre, J. Deschamps, M. Georgelin, R. Guérin, A. Pocheau, Directional solidification of inclined structures in thin samples, Acta Mater. 74 (2014) 255-267.

[55] C. A. Wulff, E. F. Westerum Jr., Heat capacities and thermodynamic properties of globular molecules. VI. Succinonitrile, J. Phys. Chem. 67 (1963) 2376-2381.

[56] B. Caroli, C. Caroli, L. Ramirez-Piscina, Initial front transients in directional solidification of thin samples of dilute alloys, J. Cryst. Growth 132 (1993) 377-388.

[57] T.F. Bower, H.D. Brody, M.C. Flemings, Measurements of solute redistribution in dendritic solidification, Trans. Met. Soc. AIME 236 (1966) 624-633

[58] A. Karma, P. Pelcé, Cell-to-dendrite transition in directional solidification, Europhys. Lett. 9 (1989) $713-718$.

[59] A. Karma, P. Pelcé, Oscillatory instability of deep cells in directional solidification, Phys. Rev. A 39 (1989) 4162-4169.

[60] P. G. Saffman, G. Taylor, The penetration of a fluid into a porous medium or Hele-Shaw cell containing a more viscous liquid, Proc. Roy. Soc. A 245 (1958) 312-329.

[61] J. W. McLean, P. G. Saffman, The effect of surface tension on the shape of fingers in a Hele Shaw cell, J. Fluid Mech. 102 (1981) 455-469

[62] R. Combescot, T. Dombre, V. Hakim, Y. Pomeau, A. Pumir, Shape selection of Saffman-Taylor Fingers, Phys. Rev. Lett. 56 (1986) 2036-2039.

[63] J.-M. Vanden Broeck, Fingers in a Hele-Shaw cell with surface tension, Phys. Fluids 26 (1983) $2033-2034$. 


\section{List of Figures}

1 Schematic figure of the experimental setup (upper figure) and temperature fields at steady state (bottom figure). The blue thin, gray dotted, and red thick lines in the bottom figure show stationary temperature profiles predicted by $\mathrm{PF}$ simulation at $V=4 \mu \mathrm{m} / \mathrm{s}$ using the FTA, TDA, and TFC (see text), respectively. . . . . . . . . . . . . . . .

2 Microstructures observed in the microgravity experiment for $V=4 \mu \mathrm{m} / \mathrm{s}$ at different solidified lengths $L=V t$. Images show the solid-liquid interface seen from the top (i.e. microstructures are growing towards the camera). Typical ridges and poxes appearing in the early stages of planar instability are illustrated in (b), together with the FFT used to identify the early stage

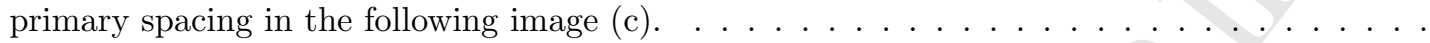

3 Average primary spacing evolution as a function of the solidified length $(L=V t)$ for different pulling velocities $V$ (a), and spacing distribution at $L=57.5 \mathrm{~mm}$ for $V=4 \mu \mathrm{m} / \mathrm{s}$ (i.e. the morphology in Fig. 2f) (b) in the DECLIC-DSI experiments. Black cross symbols in (a) correspond to the microstructures illustrated in Fig. 2c-f. . . . . . . . . . . . . .

4 Interface dynamics under different thermal effects. The interface dynamics measured in the experiment (black squares) is compared to PF simulations with different thermal considerations, i.e. FTA (blue thin), TDA (gray dotted), and TFC (red thick), for $V[\mu \mathrm{m} / \mathrm{s}]=1$ (a), 2 (b), 4 (c), and 8 (d). Red background shaded areas show time ranges for the onset of morphological instability observed in experiments. Green plus symbols in (c) relates to the microstructures in Fig. 5. . . . . . . . . . . . . . . . . . . .

5 Microstructures at different time steps (left to right) in the TFC simulation at $V=4 \mu \mathrm{m} / \mathrm{s}$, seen from the top (top row) and from the side (bottom row). . . . . . . . . . . . . . 30

6 Time evolution of the interface liquid solute concentration $c_{l}$ (a), interface velocity $V_{i}$ (b), and average spacing (c) as a function of the solidified length $L$ for different pulling velocities $V=$ 2 (left column), 4 (center column), and $8 \mu \mathrm{m} / \mathrm{s}$ (right column) in both FTA (blue thin line) and TFC (red thick line). The shaded area shows the experimentally observed time range for the onset of morphological instability. Plus symbols mark the time steps for snapshots in Fig. 5. The dashed lines in (b) relate to $V_{c}$ using the constitutional undercooling criterion Eq. (5) [39, 38] (constant black dashed line) and a time dependent $\Delta c_{i}(t)$ from planar front simulations (colored dashed lines). . . . . . . . . . . . . . . . . .

7 Initially selected spacing $\Lambda_{0}$ (a) and the measured $t_{0}, t_{c}$, and $t_{1}$ (b) as a function of pulling velocity $V$. In (a), measured average initial spacings in the experiments (black squares), and in PF simulations using the FTA (blue diamonds) and the TFC (red circles) are compared to the critical wavelength $\Lambda_{c}$. The black error bars correspond to minimum and maximum spacings in experiments. (b) shows the measured $t_{0}$ (up triangles) when $V_{i}(t)$ intersects $V_{c}(t)$, $t_{c}$ (filled circle dots) the critical time for $\Lambda_{c}$, and $t_{1}$ (down triangles) for the onset of morphological instability from the FTA (blue symbols) and the TFC (red symbols) simulations. Black vertical error bars correspond to time ranges of the morphological instability in the experiments (i.e. red shaded areas in Fig. 4 and Fig. 6). The subset of (b) using a log-log scale shows the time interval between $t_{0}$ and $t_{1}$ in the FTA (blue plus dots) and the TFC (red cross dots) as a function of $V$ with corresponding fitting lines. . . . . . . . . . . . . .

8 Steady state tip undercooling (a) and radius (b) as a function of the primary spacing, with dashed lines corresponding to spacing unstable to cell elimination. The stable spacing ranges identified for reduced system sizes in (a)-(b) are reported in (c) as rectangular boxes, and compared to dynamically selected spacings in spatially extended domains in experiments (black squares for average and error bars for standard deviations) and in simulations with different thermal representations (color symbols) as a function of the pulling velocity. The black dashed line shows a guideline $\Lambda \sim V^{-1 / 4}$. 
9 Interface shape and thermal field for $V=4 \mu \mathrm{m} / \mathrm{s}$ at different times $t[\mathrm{~s}]=$ (a) 494 (near the peak velocity), (b) 579, (c) 2011, and (d) 3000 (steady state). Contour lines show iso-values of the temperature field on a $z=z_{i}$ plane located just ahead of the most advanced tip from higher temperatures (red) $T_{\text {Max }}[\mathrm{K}]=$ (a) 330.409, (b) 330.577, (c) 330.617, and (d) 330.623 to lower temperatures (blue) with steps of $0.5 \times 10^{-3} \mathrm{~K}$. Dotted green lines in (d) show the location of a stationary trailing cell. . . . . . . . . . . . . . . . . . . 34 


\section{Figures}
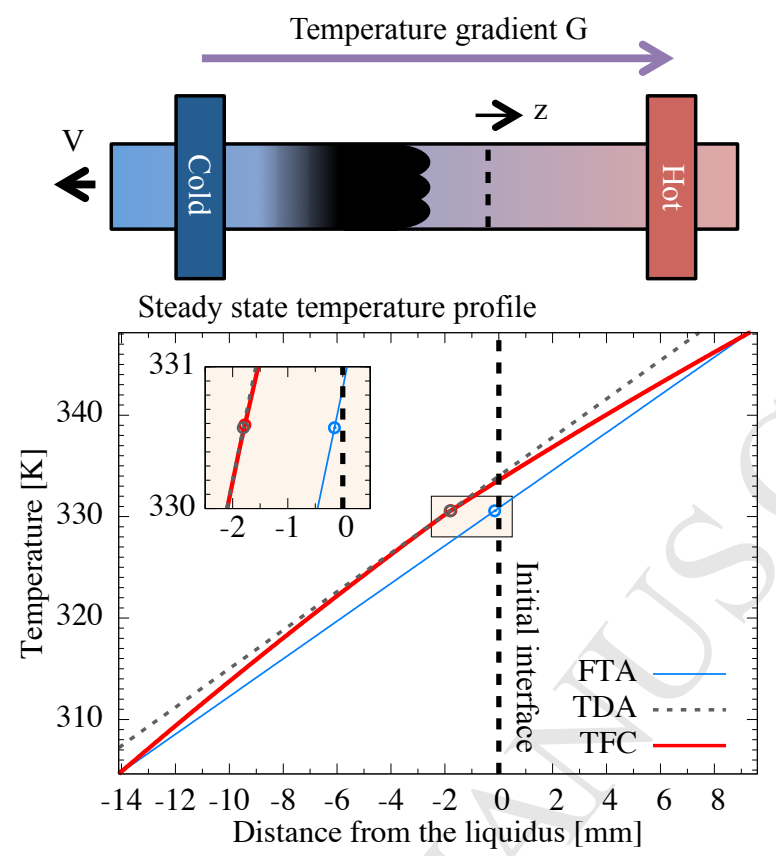

Figure 1: Schematic figure of the experimental setup (upper figure) and temperature fields at steady state (bottom figure). The blue thin, gray dotted, and red thick lines in the bottom figure show stationary temperature profiles predicted by $\mathrm{PF}$ simulation at $V=4 \mu \mathrm{m} / \mathrm{s}$ using the FTA, TDA, and TFC (see text), respectively. 
(a) $\mathrm{L}=0 \mathrm{~mm}$

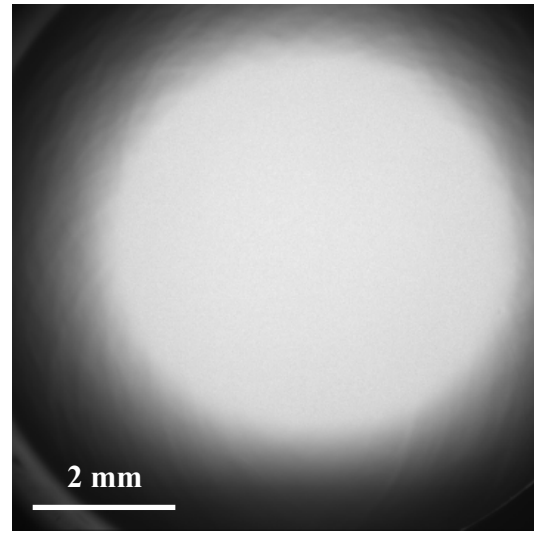

(d) $\mathrm{L}=5.02 \mathrm{~mm}$

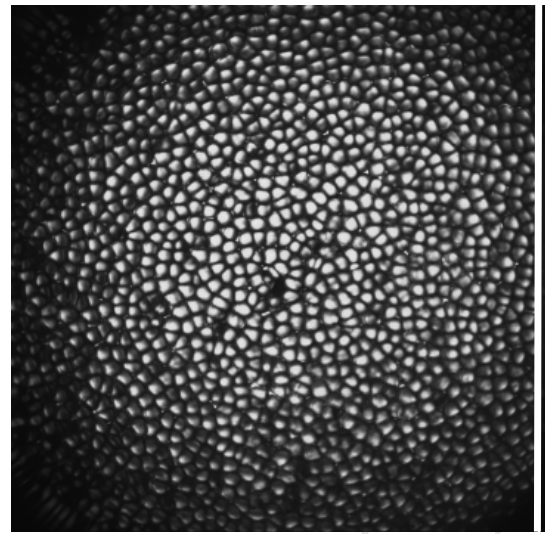

(b) $\mathrm{L}=1.77 \mathrm{~mm}$

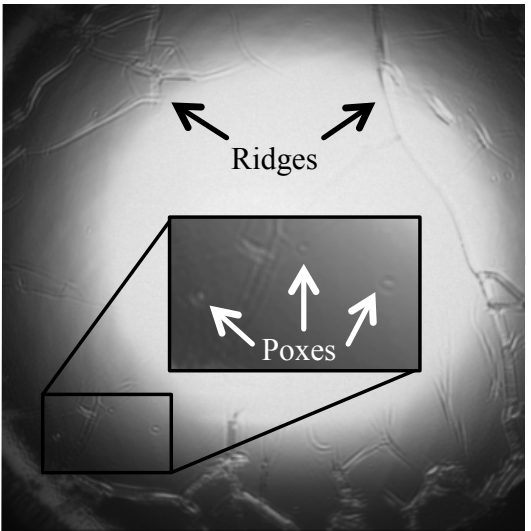

(e) $\mathrm{L}=21.8 \mathrm{~mm}$

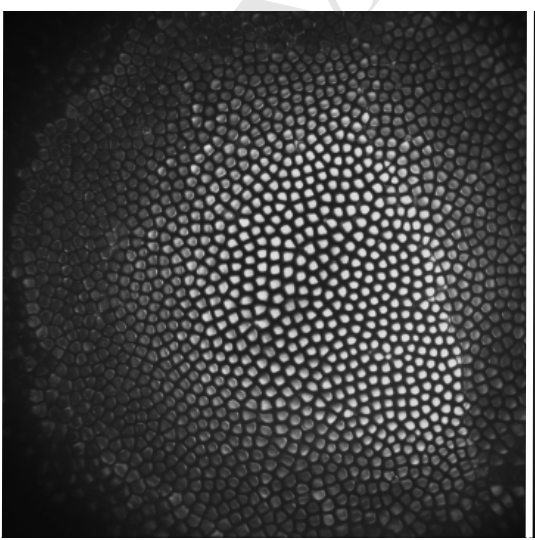

(c) $\mathrm{L}=1.96 \mathrm{~mm}$

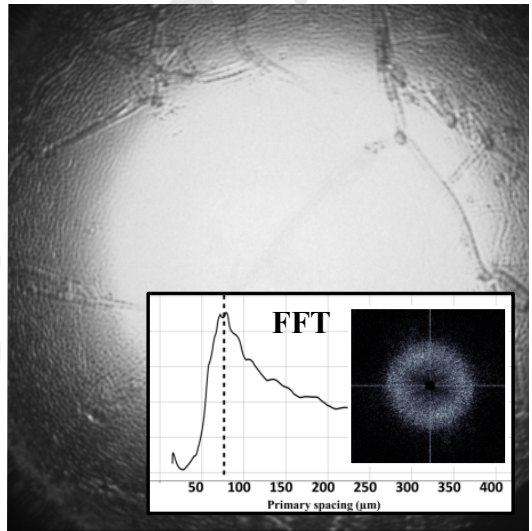

(f) $\mathrm{L}=57.5 \mathrm{~mm}$

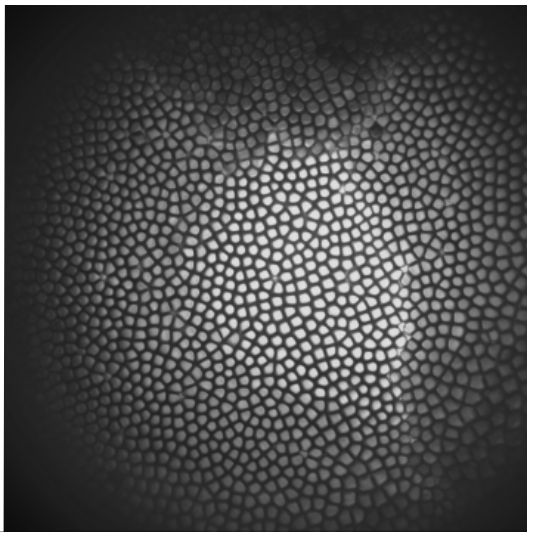

Figure 2: Microstructures observed in the microgravity experiment for $V=4 \mu \mathrm{m} / \mathrm{s}$ at different solidified lengths $L=V t$. Images show the solid-liquid interface seen from the top (i.e. microstructures are growing towards the camera). Typical ridges and poxes appearing in the early stages of planar instability are illustrated in (b), together with the FFT used to identify the early stage primary spacing in the following image (c). 
(a) Average primary spacing evolution

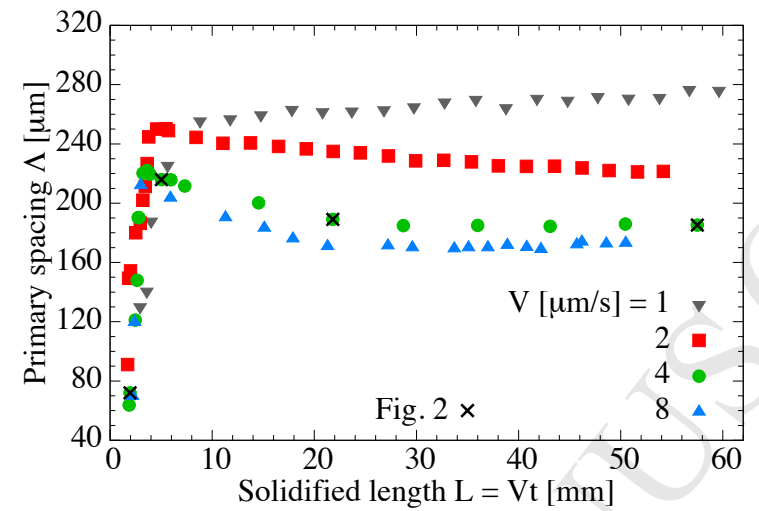

(b) Primary spacing distribution for $\mathrm{V}=4 \mu \mathrm{m} / \mathrm{s}$

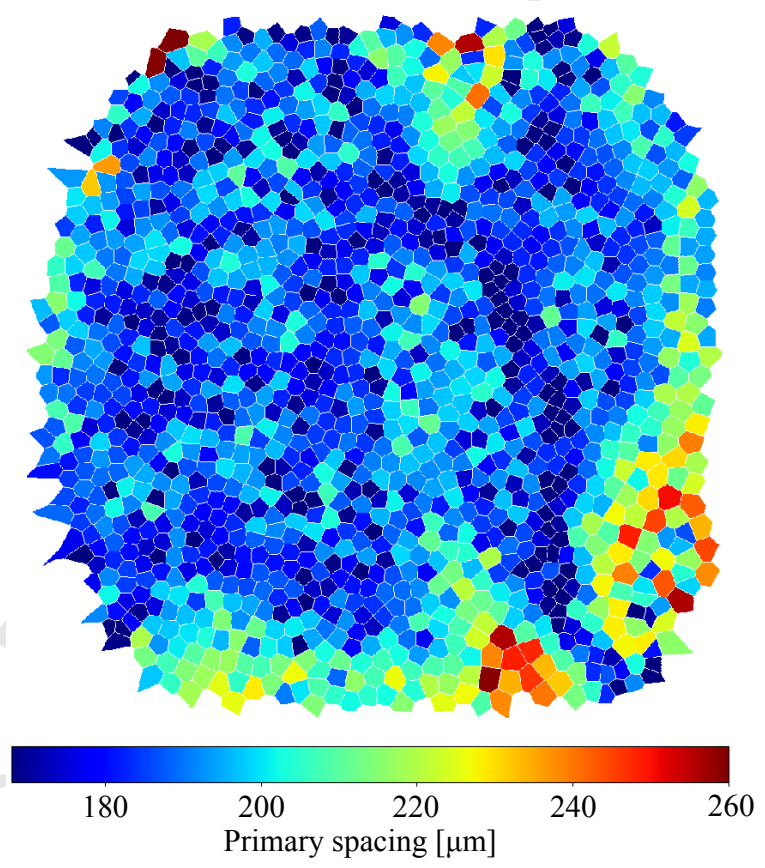

Figure 3: Average primary spacing evolution as a function of the solidified length $(L=V t)$ for different pulling velocities $V$ (a), and spacing distribution at $L=57.5 \mathrm{~mm}$ for $V=4 \mu \mathrm{m} / \mathrm{s}$ (i.e. the morphology in Fig. 2f) (b) in the DECLIC-DSI experiments. Black cross symbols in (a) correspond to the microstructures illustrated in Fig. 2c-f. 

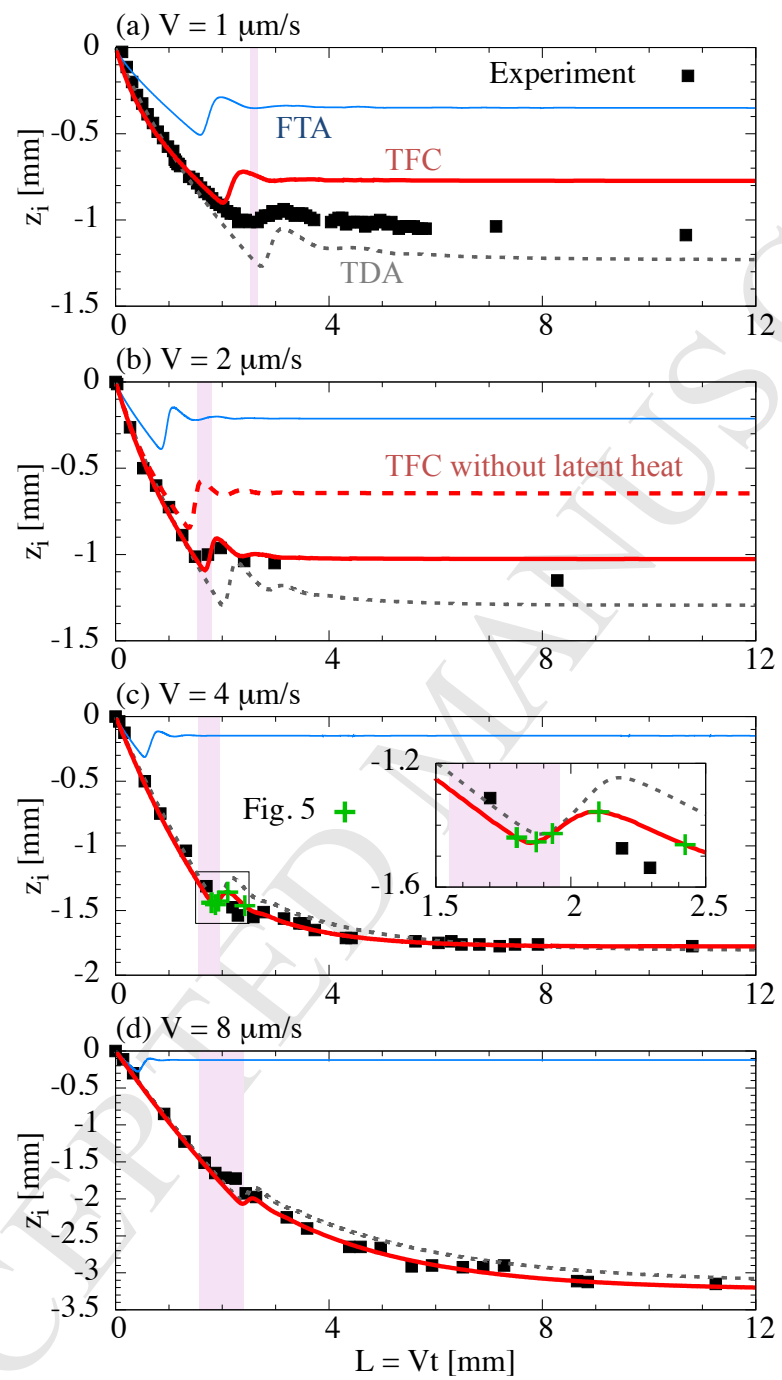

Figure 4: Interface dynamics under different thermal effects. The interface dynamics measured in the experiment (black squares) is compared to PF simulations with different thermal considerations, i.e. FTA (blue thin), TDA (gray dotted), and TFC (red thick), for $V[\mu \mathrm{m} / \mathrm{s}]=1$ (a), 2 (b), 4 (c), and 8 (d). Red background shaded areas show time ranges for the onset of morphological instability observed in experiments. Green plus symbols in (c) relates to the microstructures in Fig. 5. 
(a) $\mathrm{L}=1.80 \mathrm{~mm}$

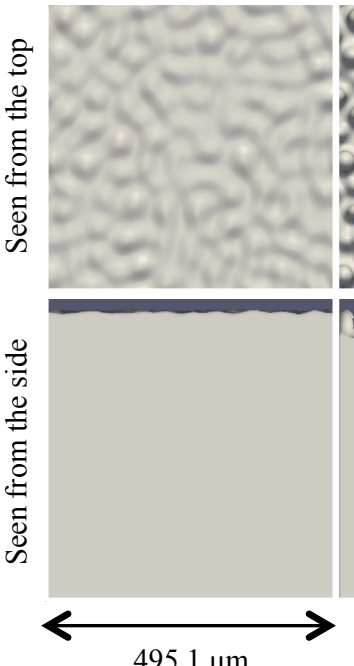

$495.1 \mu \mathrm{m}$ (b) $\mathrm{L}=1.87 \mathrm{~mm}$

(c) $\mathrm{L}=1.93 \mathrm{~mm}$
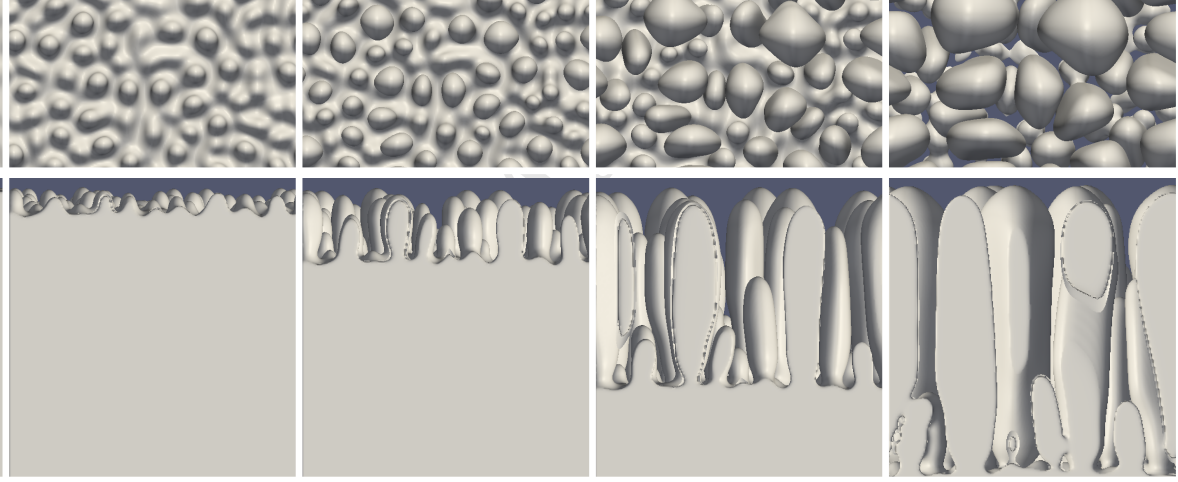

y

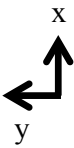

$\epsilon^{2}$

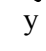

Figure 5: Microstructures at different time steps (left to right) in the TFC simulation at $V=4 \mu \mathrm{m} / \mathrm{s}$, seen from the top (top row) and from the side (bottom row). 

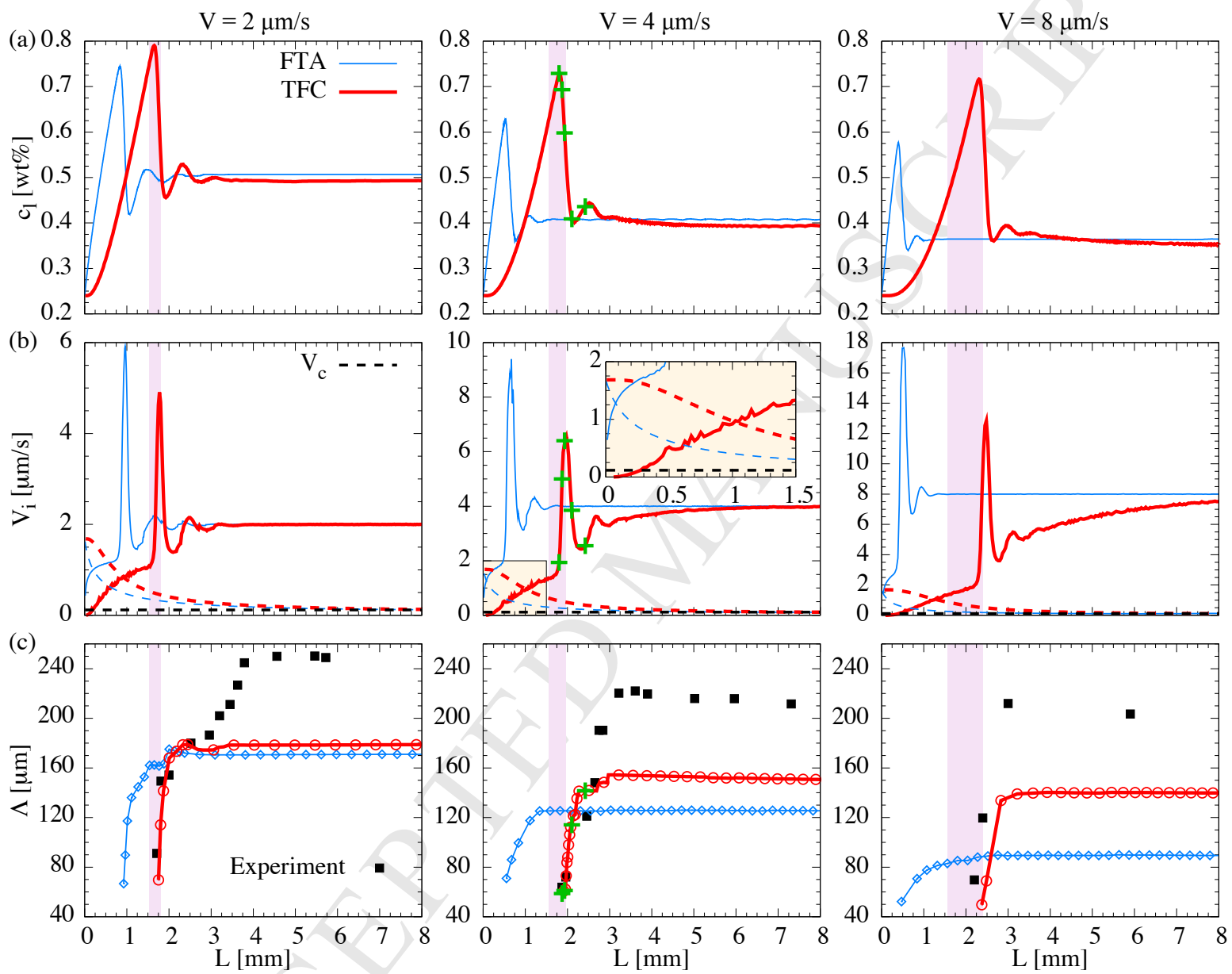

Figure 6: Time evolution of the interface liquid solute concentration $c_{l}$ (a), interface velocity $V_{i}$ (b), and average spacing (c) as a function of the solidified length $L$ for different pulling velocities $V=2$ (left column), 4 (center column), and $8 \mu \mathrm{m} / \mathrm{s}$ (right column) in both FTA (blue thin line) and TFC (red thick line). The shaded area shows the experimentally observed time range for the onset of morphological instability. Plus symbols mark the time steps for snapshots in Fig. 5. The dashed lines in (b) relate to $V_{c}$ using the constitutional undercooling criterion Eq. (5) [39, 38] (constant black dashed line) and a time dependent $\Delta c_{i}(t)$ from planar front simulations (colored dashed lines). 


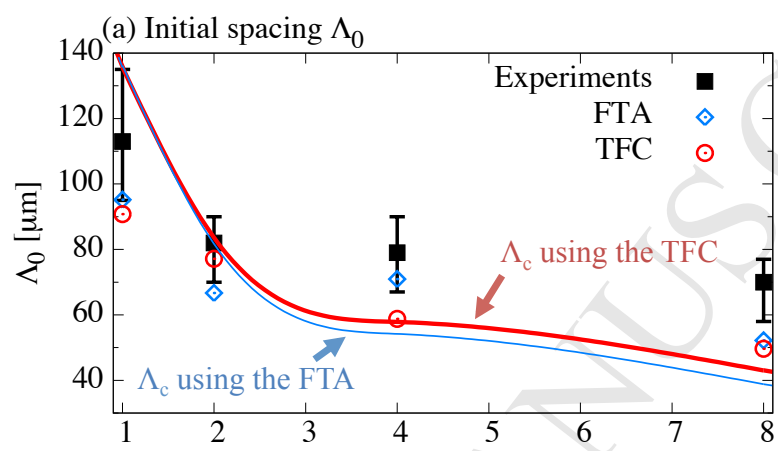

(b) Time for morphological instability

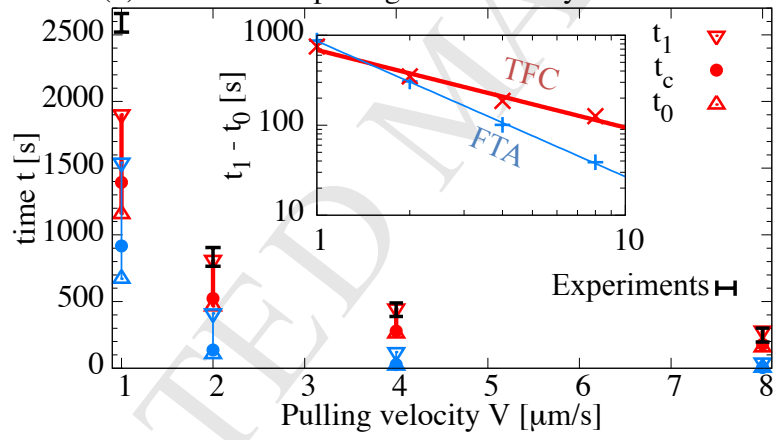

Figure 7: Initially selected spacing $\Lambda_{0}$ (a) and the measured $t_{0}, t_{c}$, and $t_{1}$ (b) as a function of pulling velocity $V$. In (a), measured average initial spacings in the experiments (black squares), and in PF simulations using the FTA (blue diamonds) and the TFC (red circles) are compared to the critical wavelength $\Lambda_{c}$. The black error bars correspond to minimum and maximum spacings in experiments. (b) shows the measured $t_{0}$ (up triangles) when $V_{i}(t)$ intersects $V_{c}(t), t_{c}$ (filled circle dots) the critical time for $\Lambda_{c}$, and $t_{1}$ (down triangles) for the onset of morphological instability from the FTA (blue symbols) and the TFC (red symbols) simulations. Black vertical error bars correspond to time ranges of the morphological instability in the experiments (i.e. red shaded areas in Fig. 4 and Fig. 6). The subset of (b) using a log-log scale shows the time interval between $t_{0}$ and $t_{1}$ in the FTA (blue plus dots) and the TFC (red cross dots) as a function of $V$ with corresponding fitting lines. 

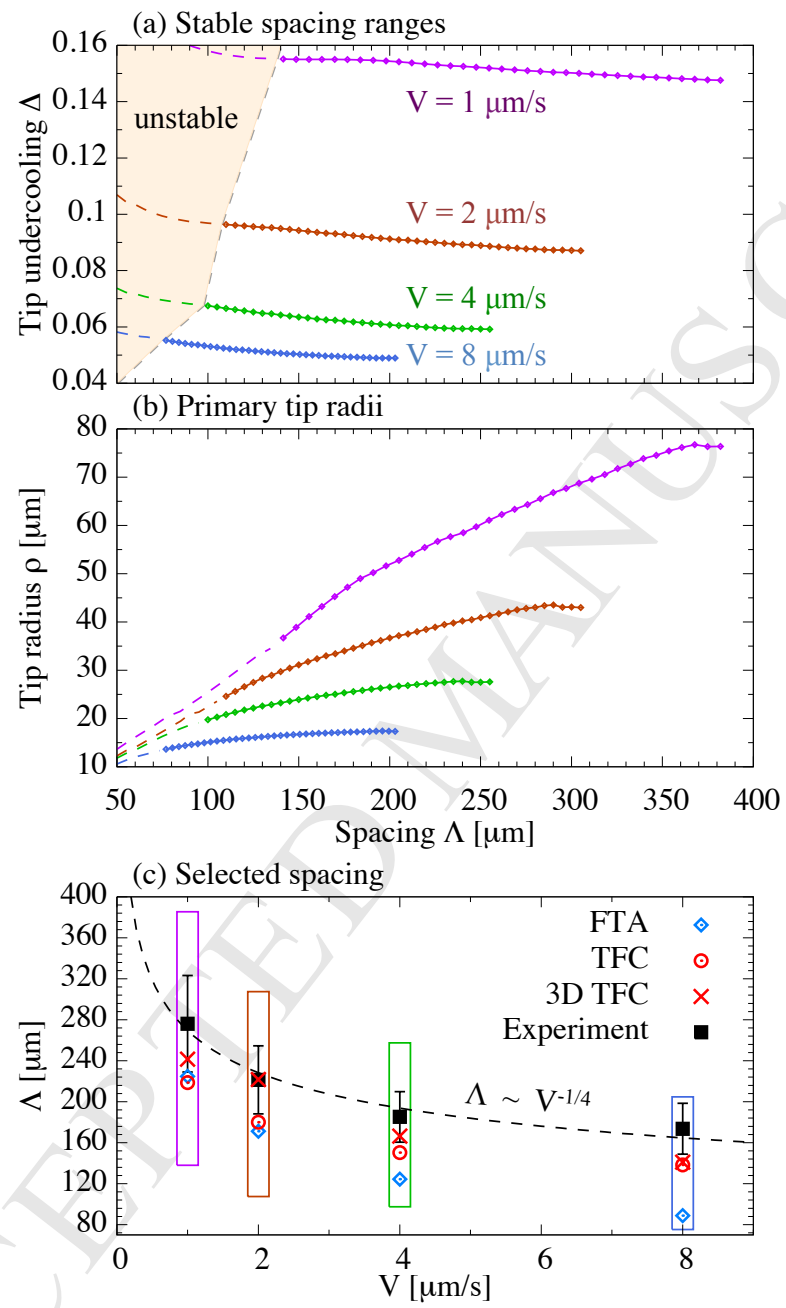

Figure 8: Steady state tip undercooling (a) and radius (b) as a function of the primary spacing, with dashed lines corresponding to spacing unstable to cell elimination. The stable spacing ranges identified for reduced system sizes in (a)-(b) are reported in (c) as rectangular boxes, and compared to dynamically selected spacings in spatially extended domains in experiments (black squares for average and error bars for standard deviations) and in simulations with different thermal representations (color symbols) as a function of the pulling velocity. The black dashed line shows a guideline $\Lambda \sim V^{-1 / 4}$. 


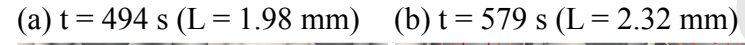

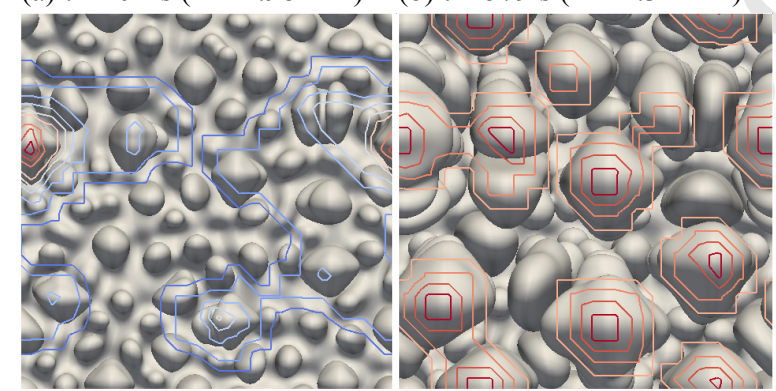

(c) $\mathrm{t}=2011 \mathrm{~s}(\mathrm{~L}=8.04 \mathrm{~mm})(\mathrm{d}) \mathrm{t}=3000 \mathrm{~s}(\mathrm{~L}=12.0 \mathrm{~mm})$

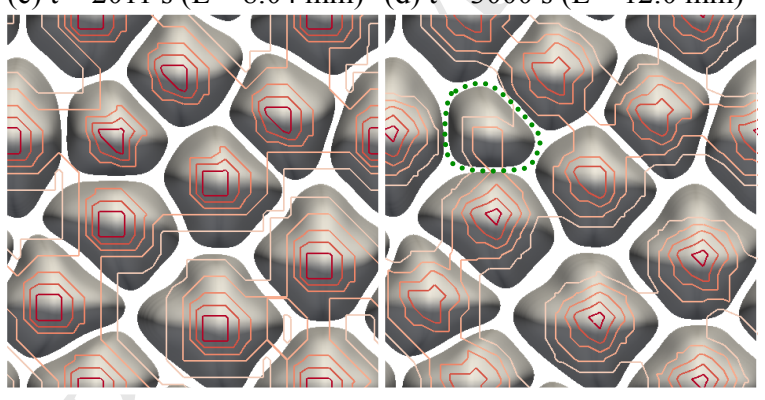

Figure 9: Interface shape and thermal field for $V=4 \mu \mathrm{m} / \mathrm{s}$ at different times $t$ [s] $=$ (a) 494 (near the peak velocity), (b) 579 , (c) 2011, and (d) 3000 (steady state). Contour lines show iso-values of the temperature field on a $z=z_{i}$ plane located just ahead of the most advanced tip from higher temperatures (red) $T_{M a x}[\mathrm{~K}]=$ (a) 330.409, (b) 330.577, (c) 330.617, and (d) 330.623 to lower temperatures (blue) with steps of $0.5 \times 10^{-3} \mathrm{~K}$. Dotted green lines in (d) show the location of a stationary trailing cell. 


\section{List of Tables}

1 Parameters for the thermal drift approximation at $G=19 \mathrm{~K} / \mathrm{cm}$. The corresponding alloy thermal length is $l_{T} \approx 2.29 \mathrm{~mm}$. The dimensionless tip undercooling $\Delta$, used to calculate the total isotherm shift $\Delta z_{T}$, comes from PF simulations of a quarter of a cell approximately at the experimentally measured spacing (Fig. 8a) . . . . . . . . . . . . . . . . . . 36

2 Stationary state tip undercooling from phase-field calculations using the TFC $\left(\Delta_{\mathrm{PF}}\right)$, compared to predictions of the Karma-Pelcé model [59] $\left(\Delta_{\mathrm{KP}}\right)$, and the Bower-Brody-Flemings

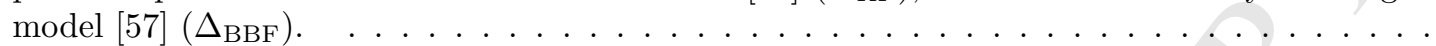




\section{Tables}

Table 1: Parameters for the thermal drift approximation at $G=19 \mathrm{~K} / \mathrm{cm}$. The corresponding alloy thermal length is $l_{T} \approx$ $2.29 \mathrm{~mm}$. The dimensionless tip undercooling $\Delta$, used to calculate the total isotherm shift $\Delta z_{T}$, comes from PF simulations of a quarter of a cell approximately at the experimentally measured spacing (Fig. 8a).

\begin{tabular}{ccccc}
\hline $\begin{array}{c}V \\
{[\mu \mathrm{m} / \mathrm{s}]}\end{array}$ & $\begin{array}{c}\Delta z_{T} \\
{[\mathrm{~mm}]}\end{array}$ & $\begin{array}{c}\Delta z_{\exp } \\
{[\mathrm{mm}]}\end{array}$ & $\Delta$ & $\begin{array}{c}\tau_{d} \\
{[\mathrm{~s}]}\end{array}$ \\
\hline \hline 1 & 0.88 & 1.23 & 0.152 & 1778 \\
2 & 1.08 & 1.29 & 0.090 & 688 \\
4 & 1.66 & 1.80 & 0.061 & 491 \\
8 & 3.02 & 3.13 & 0.050 & 378 \\
\hline
\end{tabular}


Table 2: Stationary state tip undercooling from phase-field calculations using the TFC $\left(\Delta_{\mathrm{PF}}\right)$, compared to predictions of the Karma-Pelcé model [59] ( $\left.\Delta_{\mathrm{KP}}\right)$, and the Bower-Brody-Flemings model [57] $\left(\Delta_{\mathrm{BBF}}\right)$.

\begin{tabular}{cccc}
\hline $\mathrm{V}[\mu \mathrm{m} / \mathrm{s}]$ & $\Delta_{\mathrm{PF}}$ & $\Delta_{\mathrm{KP}}$ & $\Delta_{\mathrm{BBF}}$ \\
\hline \hline 1 & 0.148 & 0.138 & 0.118 \\
2 & 0.0882 & 0.0804 & 0.0589 \\
4 & 0.0593 & 0.0516 & 0.0295 \\
8 & 0.0489 & 0.0372 & 0.0147 \\
\hline
\end{tabular}




\section{List of Tables}

1 Parameters for the thermal drift approximation at $G=19 \mathrm{~K} / \mathrm{cm}$. The corresponding alloy thermal length is $l_{T} \approx 2.29 \mathrm{~mm}$. The dimensionless tip undercooling $\Delta$, used to calculate the total isotherm shift $\Delta z_{T}$, comes from $\mathrm{PF}$ simulations of a quarter of a cell approximately at the experimentally measured spacing (Fig. 8a) . . . . . . . . . . . . . . . . . 36

2 Stationary state tip undercooling from phase-field calculations using the TFC $\left(\Delta_{\mathrm{PF}}\right)$, compared to predictions of the Karma-Pelcé model [59] $\left(\Delta_{\mathrm{KP}}\right)$, and the Bower-Brody-Flemings model $[57]\left(\Delta_{\mathrm{BBF}}\right) . \ldots \ldots \ldots \ldots \ldots \ldots \ldots \ldots \ldots$ 


\section{Tables}

Table 1: Parameters for the thermal drift approximation at $G=19 \mathrm{~K} / \mathrm{cm}$. The corresponding alloy thermal length is $l_{T} \approx$ $2.29 \mathrm{~mm}$. The dimensionless tip undercooling $\Delta$, used to calculate the total isotherm shift $\Delta z_{T}$, comes from PF simulations of a quarter of a cell approximately at the experimentally measured spacing (Fig. 8a).

\begin{tabular}{ccccc}
\hline $\begin{array}{c}V \\
{[\mu \mathrm{m} / \mathrm{s}]}\end{array}$ & $\begin{array}{c}\Delta z_{T} \\
{[\mathrm{~mm}]}\end{array}$ & $\begin{array}{c}\Delta z_{\exp } \\
{[\mathrm{mm}]}\end{array}$ & $\Delta$ & $\begin{array}{c}\tau_{d} \\
{[\mathrm{~s}]}\end{array}$ \\
\hline \hline 1 & 0.88 & 1.23 & 0.152 & 1778 \\
2 & 1.08 & 1.29 & 0.090 & 688 \\
4 & 1.66 & 1.80 & 0.061 & 491 \\
8 & 3.02 & 3.13 & 0.050 & 378 \\
\hline
\end{tabular}


Table 2: Stationary state tip undercooling from phase-field calculations using the TFC $\left(\Delta_{\mathrm{PF}}\right)$, compared to predictions of the Karma-Pelcé model [59] $\left(\Delta_{\mathrm{KP}}\right)$, and the Bower-Brody-Flemings model [57] $\left(\Delta_{\mathrm{BBF}}\right)$.

\begin{tabular}{cccc}
\hline $\mathrm{V}[\mu \mathrm{m} / \mathrm{s}]$ & $\Delta_{\mathrm{PF}}$ & $\Delta_{\mathrm{KP}}$ & $\Delta_{\mathrm{BBF}}$ \\
\hline \hline 1 & 0.148 & 0.138 & 0.118 \\
2 & 0.0882 & 0.0804 & 0.0589 \\
4 & 0.0593 & 0.0516 & 0.0295 \\
8 & 0.0489 & 0.0372 & 0.0147 \\
\hline
\end{tabular}


Temperature gradient $\mathrm{G}$

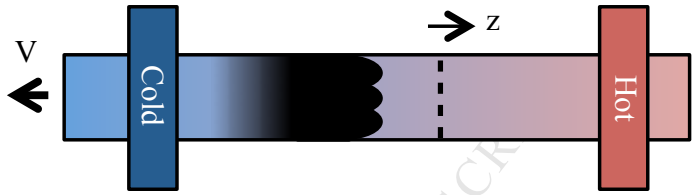

Steady state temperature profile

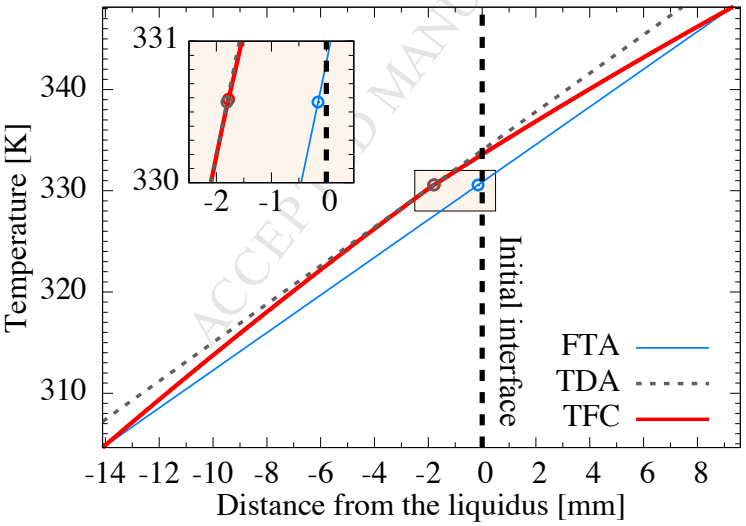


(a) Average primary spacing evolution

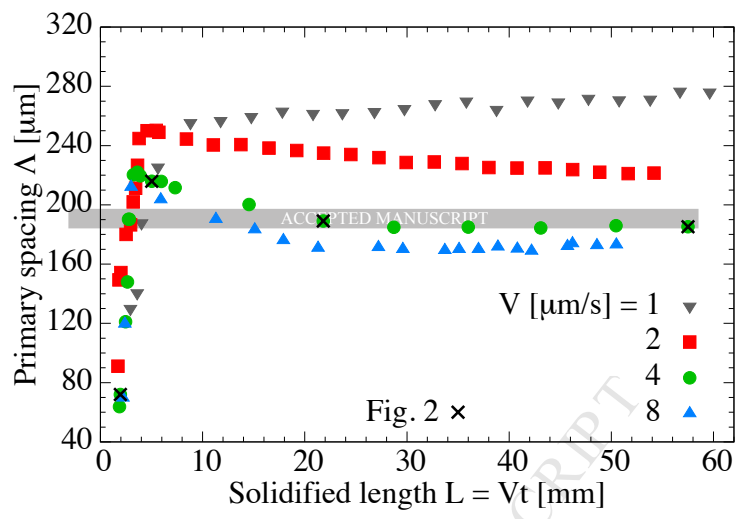

(b) Primary spacing distribution for $\mathrm{V}=4 \mu \mathrm{m} / \mathrm{s}$

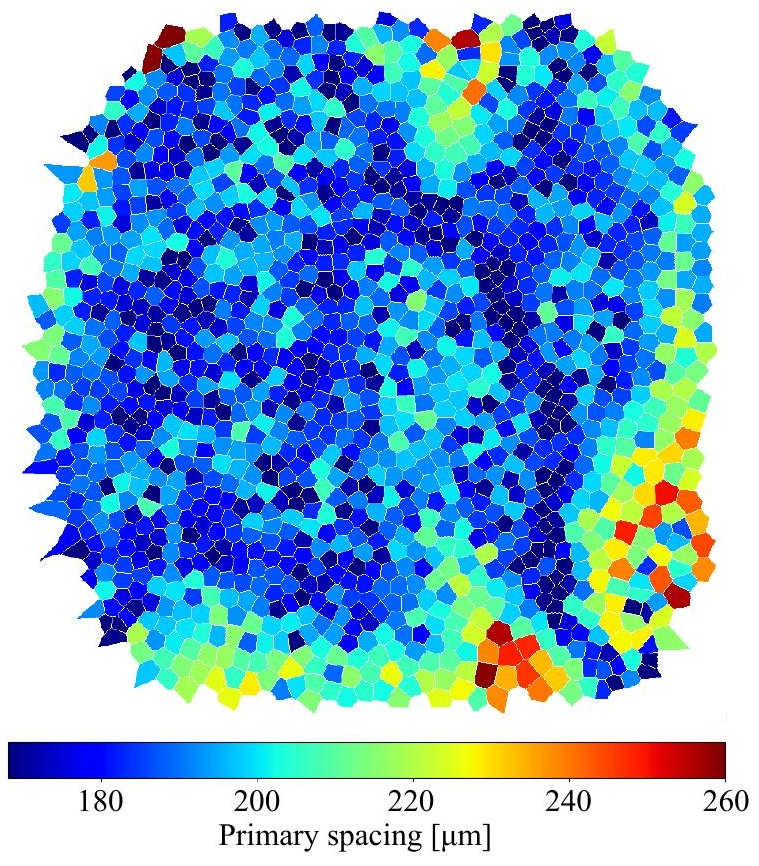


(a) $\mathrm{L}=1.80 \mathrm{~mm}$

(b) $\mathrm{L}=1.87 \mathrm{~mm}$

(c) $\mathrm{L}=1.93 \mathrm{~mm}$

(d) $\mathrm{L}=2.10 \mathrm{~mm}$

(e) $\mathrm{L}=2.42 \mathrm{~mm}$

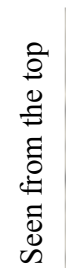

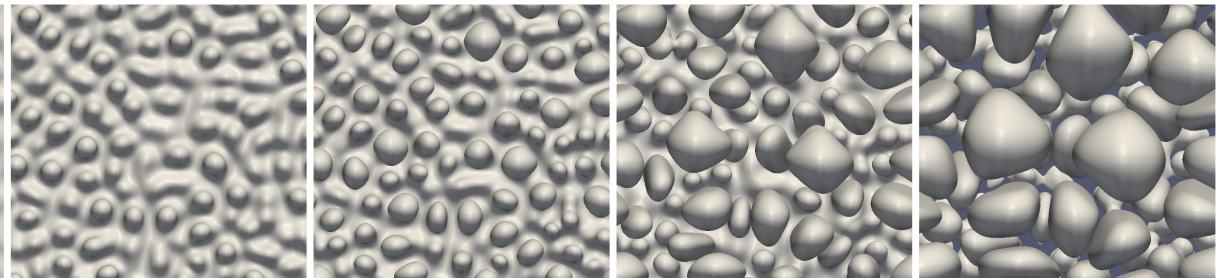

1.9.
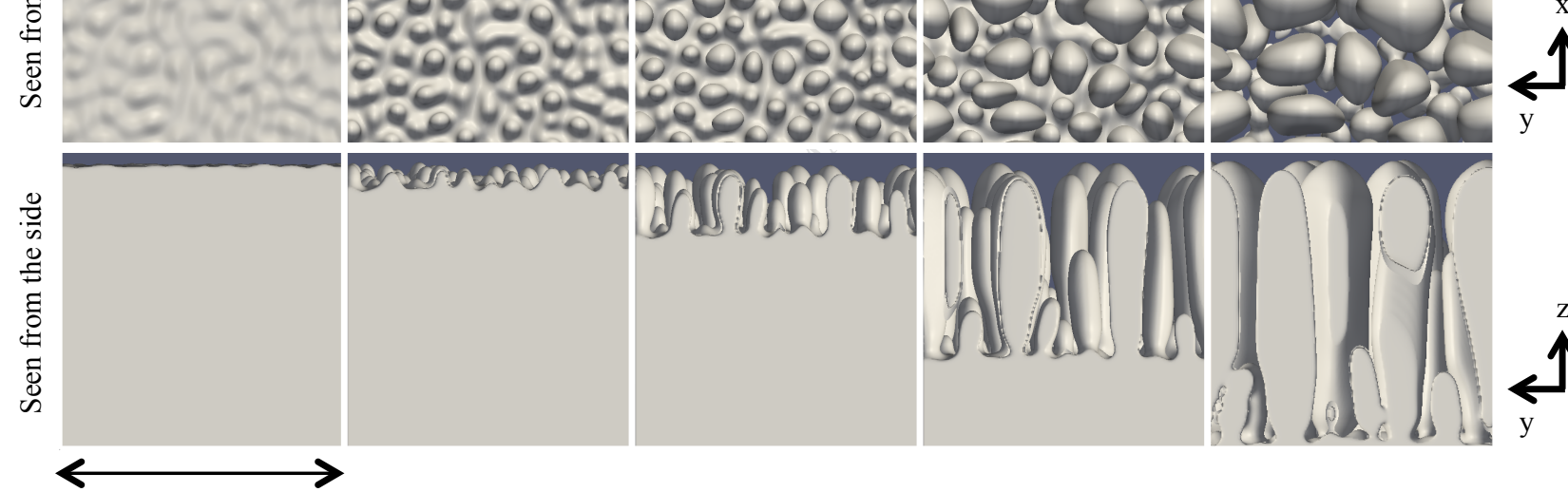

2.

$495.1 \mu \mathrm{m}$

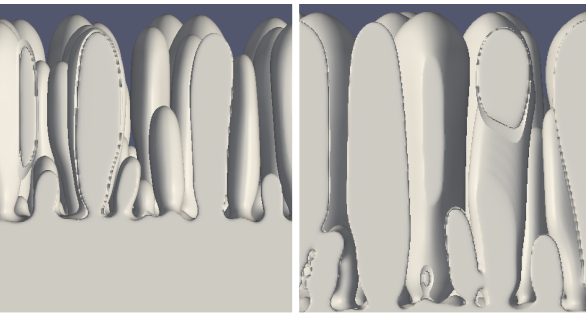

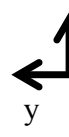




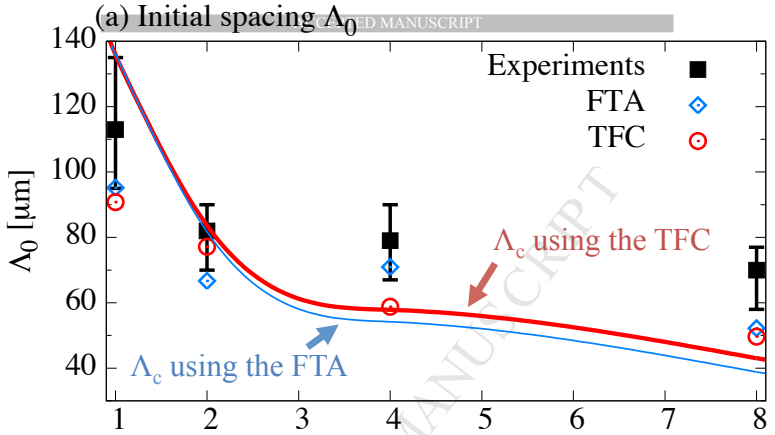

(b) Time for morphological instability

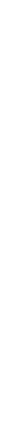


(a) Stable spacing ranges

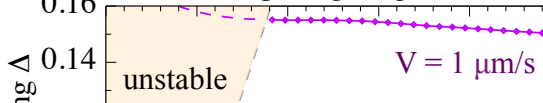

os unstable

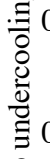

$\because 0.06$

$\mathrm{V}=2 \mu \mathrm{m} / \mathrm{s}$

0.04

(b) Primary tip radii

$$
\mathrm{V}=4 \mu \mathrm{m} / \mathrm{s}
$$

$\mathrm{V}=8 \mu \mathrm{m} / \mathrm{s}$
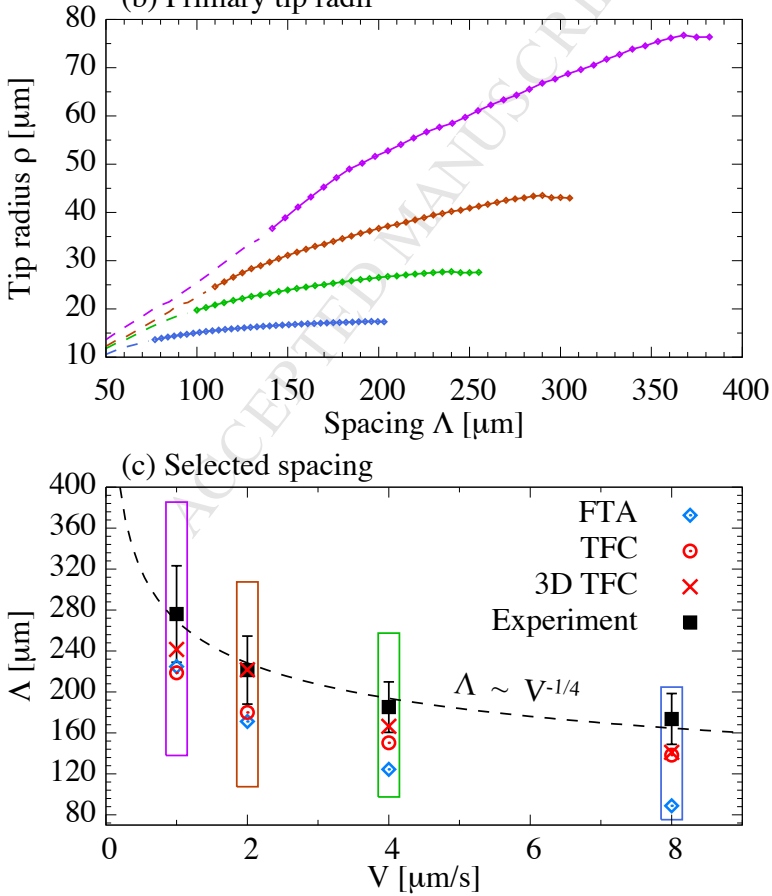
(a) $\mathrm{t}=494 \mathrm{~s}(\mathrm{~L}=1.98 \mathrm{~mm})$

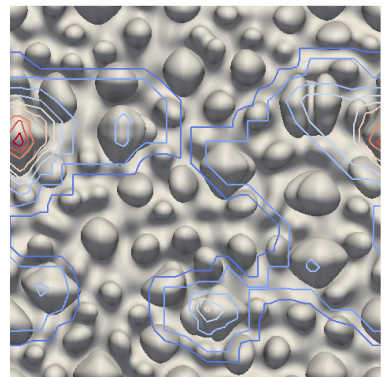

(b) $t=579 \mathrm{~s}(\mathrm{~L}=2.32 \mathrm{~mm})$

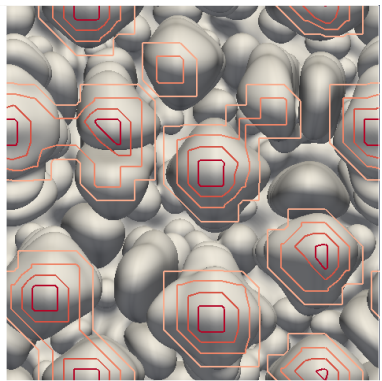

(d) $\mathrm{t}=3000 \mathrm{~s}(\mathrm{~L}=12.0 \mathrm{~mm})$ V
(c)
(c) $\mathrm{t}=2011 \mathrm{~s}(\mathrm{~L}=8.04 \mathrm{~mm})$
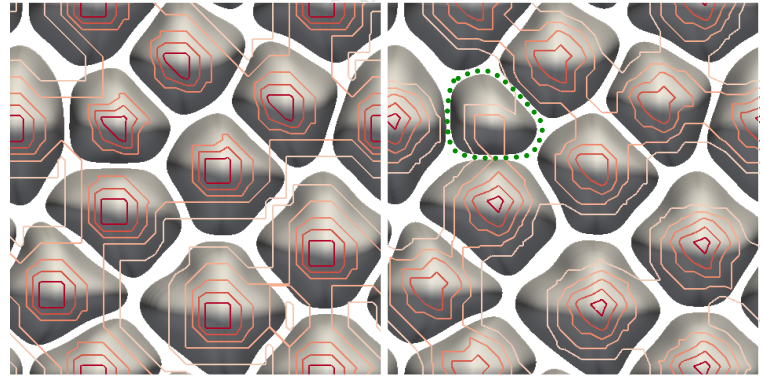


\section{List of Figures}

1 Schematic figure of the experimental setup (upper figure) and temperature fields at steady state (bottom figure). The blue thin, gray dotted, and red thick lines in the bottom figure show stationary temperature profiles predicted by $\mathrm{PF}$ simulation at $V=4 \mu \mathrm{m} / \mathrm{s}$ using the

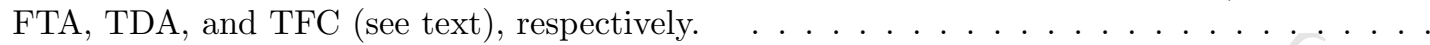

2 Microstructures observed in the microgravity experiment for $V=4 \mu \mathrm{m} / \mathrm{s}$ at different solidified lengths $L=V t$. Images show the solid-liquid interface seen from the top (i.e. microstructures are growing towards the camera). Typical ridges and poxes appearing in the early stages of planar instability are illustrated in (b), together with the FFT used to identify the early stage primary spacing in the following image $(\mathrm{c}) . \ldots \ldots \ldots \ldots \ldots$

3 Average primary spacing evolution as a function of the solidified length $(L=V t)$ for different pulling velocities $V$ (a), and spacing distribution at $L=57.5 \mathrm{~mm}$ for $V=4 \mu \mathrm{m} / \mathrm{s}$ (i.e. the morphology in Fig. 2f) (b) in the DECLIC-DSI experiments. Black cross symbols in (a) correspond to the microstructures illustrated in Fig. 2c-f. . . . . . . . . . . . . .

4 Interface dynamics under different thermal effects. The interface dynamics measured in the experiment (black squares) is compared to PF simulations with different thermal considerations, i.e. FTA (blue thin), TDA (gray dotted), and TFC (red thick), for $V[\mu \mathrm{m} / \mathrm{s}]=1$ (a), 2 (b), 4 (c), and 8 (d). Red background shaded areas show time ranges for the onset of morphological instability observed in experiments. Green plus symbols in (c) relates to the microstructures in Fig. 5. . . . . . . . . . . . . . . . . . . .

5 Microstructures at different time steps (left to right) in the TFC simulation at $V=4 \mu \mathrm{m} / \mathrm{s}$, seen from the top (top row) and from the side (bottom row). . . . . . . . . . . . . . 30

6 Time evolution of the interface liquid solute concentration $c_{l}$ (a), interface velocity $V_{i}(\mathrm{~b})$, and average spacing (c) as a function of the solidified length $L$ for different pulling velocities $V=$ 2 (left column), 4 (center column), and $8 \mu \mathrm{m} / \mathrm{s}$ (right column) in both FTA (blue thin line) and TFC (red thick line). The shaded area shows the experimentally observed time range for the onset of morphological instability. Plus symbols mark the time steps for snapshots in Fig. 5. The dashed lines in (b) relate to $V_{c}$ using the constitutional undercooling criterion Eq. (5) [39, 38] (constant black dashed line) and a time dependent $\Delta c_{i}(t)$ from planar front simulations (colored dashed lines). . . . . . . . . . . . . . . . . .

$7 \quad$ Initially selected spacing $\Lambda_{0}$ (a) and the measured $t_{0}, t_{c}$, and $t_{1}$ (b) as a function of pulling velocity $V$. In (a), measured average initial spacings in the experiments (black squares), and in PF simulations using the FTA (blue diamonds) and the TFC (red circles) are compared to the critical wavelength $\Lambda_{c}$. The black error bars correspond to minimum and maximum spacings in experiments. (b) shows the measured $t_{0}$ (up triangles) when $V_{i}(t)$ intersects $V_{c}(t)$, $t_{c}$ (filled circle dots) the critical time for $\Lambda_{c}$, and $t_{1}$ (down triangles) for the onset of morphological instability from the FTA (blue symbols) and the TFC (red symbols) simulations. Black vertical error bars correspond to time ranges of the morphological instability in the experiments (i.e. red shaded areas in Fig. 4 and Fig. 6). The subset of (b) using a log-log scale shows the time interval between $t_{0}$ and $t_{1}$ in the FTA (blue plus dots) and the TFC (red cross dots) as a function of $V$ with corresponding fitting lines. . . . . . . . . . . . . .

8 Steady state tip undercooling (a) and radius (b) as a function of the primary spacing, with dashed lines corresponding to spacing unstable to cell elimination. The stable spacing ranges identified for reduced system sizes in (a)-(b) are reported in (c) as rectangular boxes, and compared to dynamically selected spacings in spatially extended domains in experiments (black squares for average and error bars for standard deviations) and in simulations with different thermal representations (color symbols) as a function of the pulling velocity. The black dashed line shows a guideline $\Lambda \sim V^{-1 / 4}$. 
9 Interface shape and thermal field for $V=4 \mu \mathrm{m} / \mathrm{s}$ at different times $t[\mathrm{~s}]=$ (a) 494 (near the peak velocity), (b) 579, (c) 2011, and (d) 3000 (steady state). Contour lines show iso-values of the temperature field on a $z=z_{i}$ plane located just ahead of the most advanced tip from higher temperatures (red) $T_{\text {Max }}[\mathrm{K}]=$ (a) 330.409, (b) 330.577, (c) 330.617, and (d) 330.623 to lower temperatures (blue) with steps of $0.5 \times 10^{-3} \mathrm{~K}$. Dotted green lines in $(\mathrm{d})$ show the location of a stationary trailing cell. . . . . . . . . . . . . . . . . . . 34 


\section{Figures}
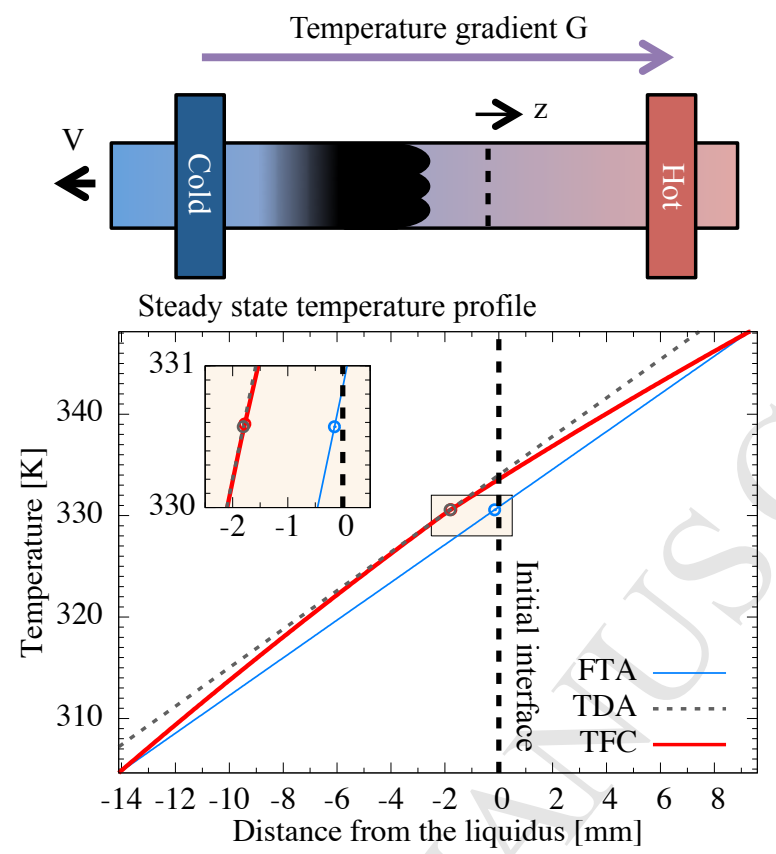

Figure 1: Schematic figure of the experimental setup (upper figure) and temperature fields at steady state (bottom figure). The blue thin, gray dotted, and red thick lines in the bottom figure show stationary temperature profiles predicted by $\mathrm{PF}$ simulation at $V=4 \mu \mathrm{m} / \mathrm{s}$ using the FTA, TDA, and TFC (see text), respectively. 
(a) $\mathrm{L}=0 \mathrm{~mm}$

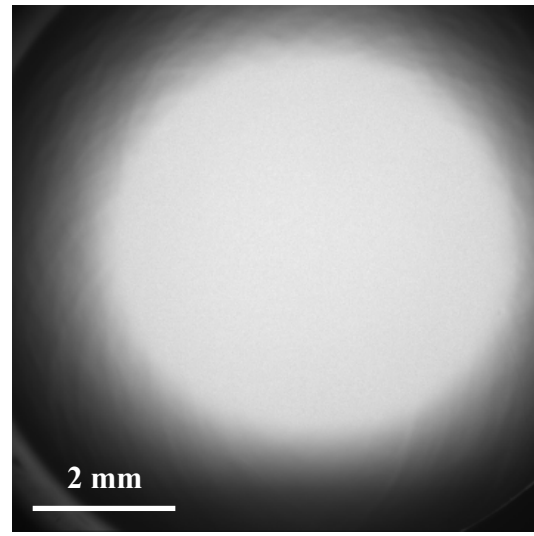

(d) $\mathrm{L}=5.02 \mathrm{~mm}$

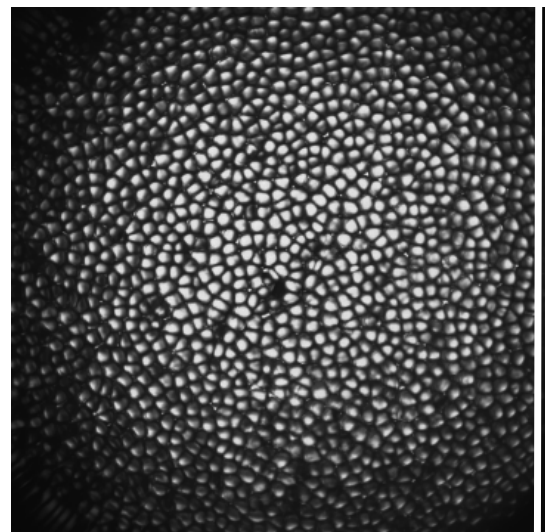

(b) $\mathrm{L}=1.77 \mathrm{~mm}$

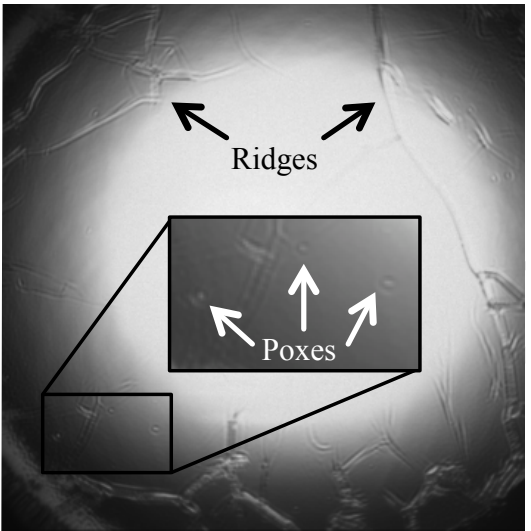

(e) $\mathrm{L}=21.8 \mathrm{~mm}$

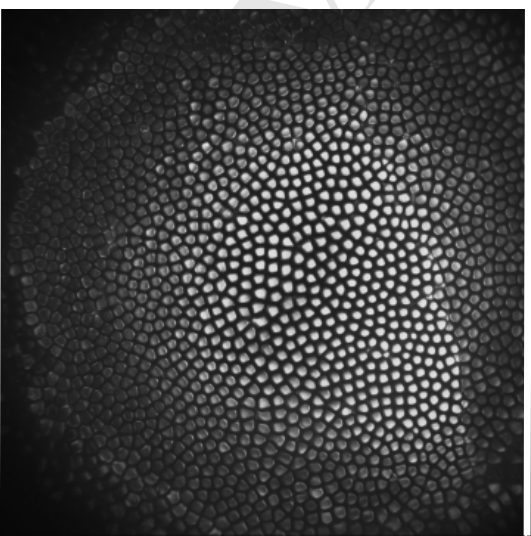

(c) $\mathrm{L}=1.96 \mathrm{~mm}$

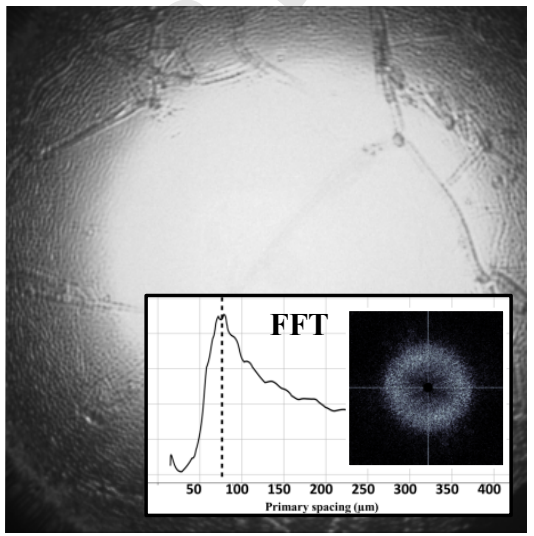

(f) $\mathrm{L}=57.5 \mathrm{~mm}$

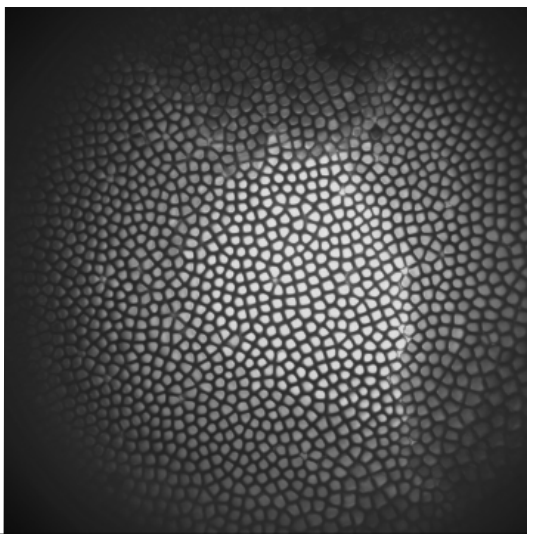

Figure 2: Microstructures observed in the microgravity experiment for $V=4 \mu \mathrm{m} / \mathrm{s}$ at different solidified lengths $L=V t$. Images show the solid-liquid interface seen from the top (i.e. microstructures are growing towards the camera). Typical ridges and poxes appearing in the early stages of planar instability are illustrated in (b), together with the FFT used to identify the early stage primary spacing in the following image (c). 
(a) Average primary spacing evolution

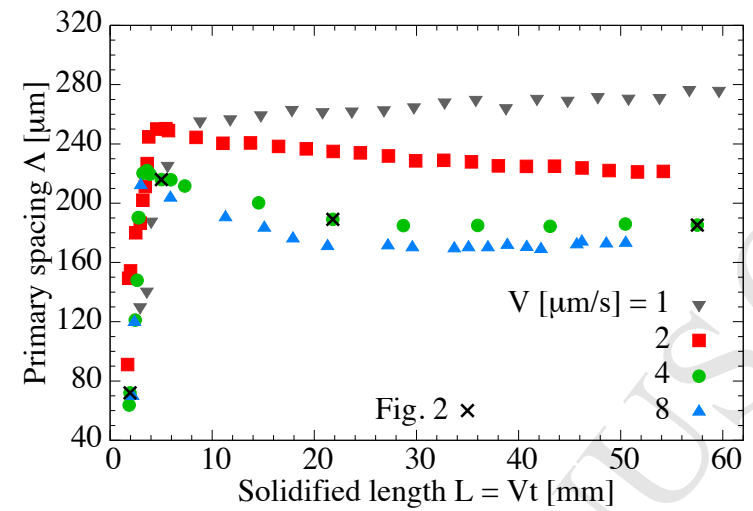

(b) Primary spacing distribution for $\mathrm{V}=4 \mu \mathrm{m} / \mathrm{s}$

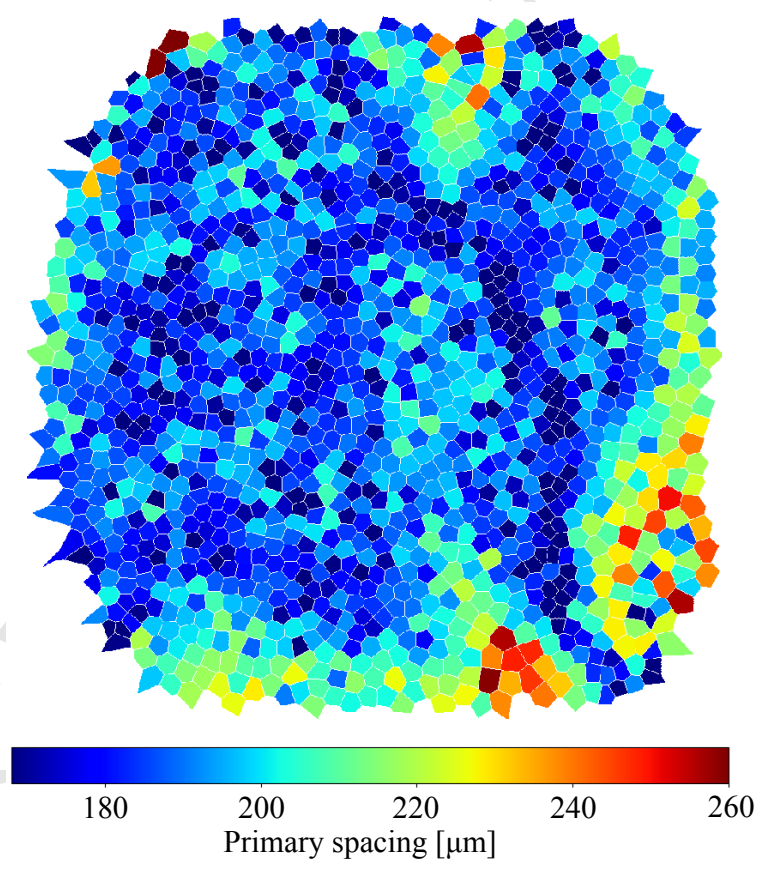

Figure 3: Average primary spacing evolution as a function of the solidified length $(L=V t)$ for different pulling velocities $V$ (a), and spacing distribution at $L=57.5 \mathrm{~mm}$ for $V=4 \mu \mathrm{m} / \mathrm{s}$ (i.e. the morphology in Fig. 2f) (b) in the DECLIC-DSI experiments. Black cross symbols in (a) correspond to the microstructures illustrated in Fig. 2c-f. 

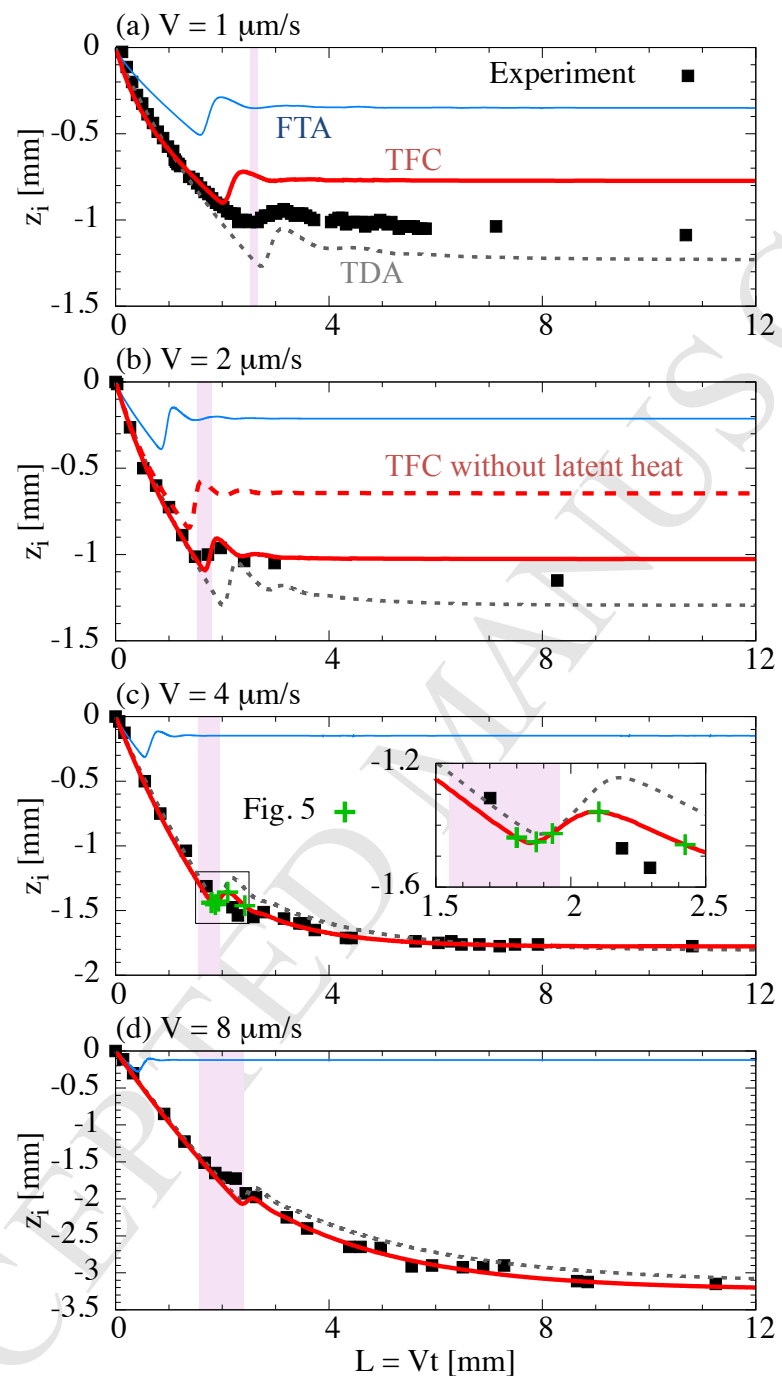

Figure 4: Interface dynamics under different thermal effects. The interface dynamics measured in the experiment (black squares) is compared to PF simulations with different thermal considerations, i.e. FTA (blue thin), TDA (gray dotted), and TFC (red thick), for $V[\mu \mathrm{m} / \mathrm{s}]=1$ (a), 2 (b), 4 (c), and 8 (d). Red background shaded areas show time ranges for the onset of morphological instability observed in experiments. Green plus symbols in (c) relates to the microstructures in Fig. 5 . 
(a) $\mathrm{L}=1.80 \mathrm{~mm}$

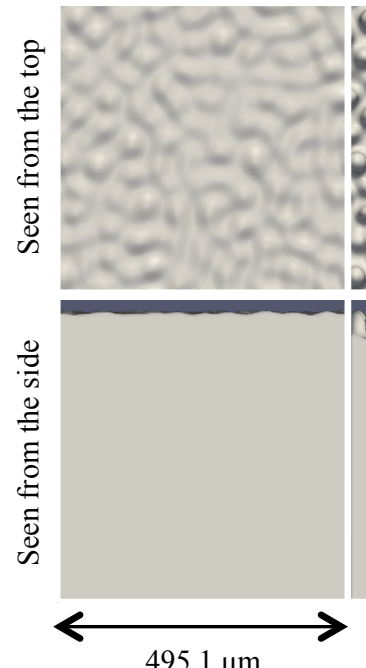

$495.1 \mu \mathrm{m}$ (b) $\mathrm{L}=1.87 \mathrm{~mm}$

(c) $\mathrm{L}=1.93 \mathrm{~mm}$

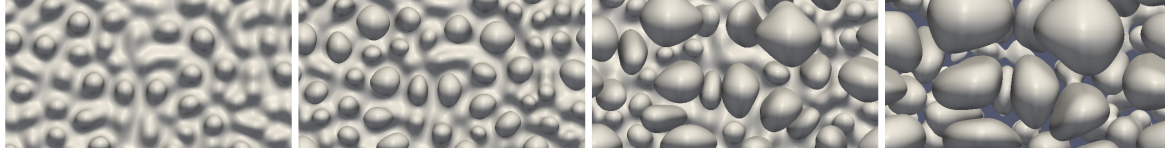

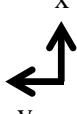
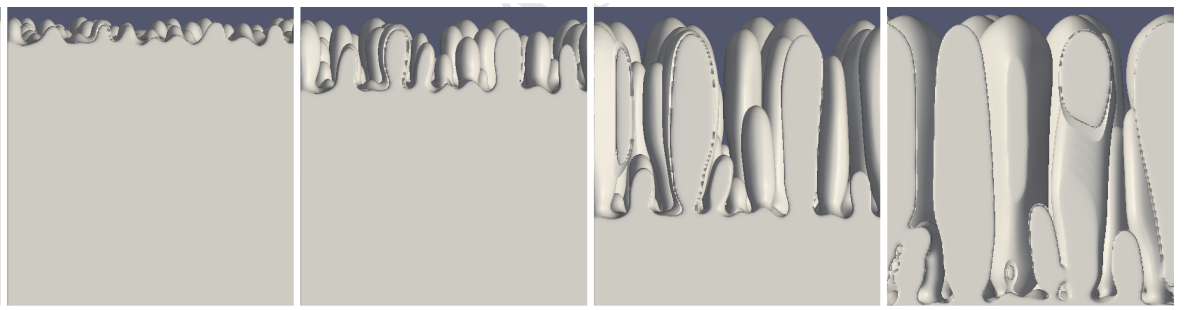

$\mathrm{y}$

$e^{2}$

Figure 5: Microstructures at different time steps (left to right) in the TFC simulation at $V=4 \mu \mathrm{m} / \mathrm{s}$, seen from the top (top row) and from the side (bottom row). 

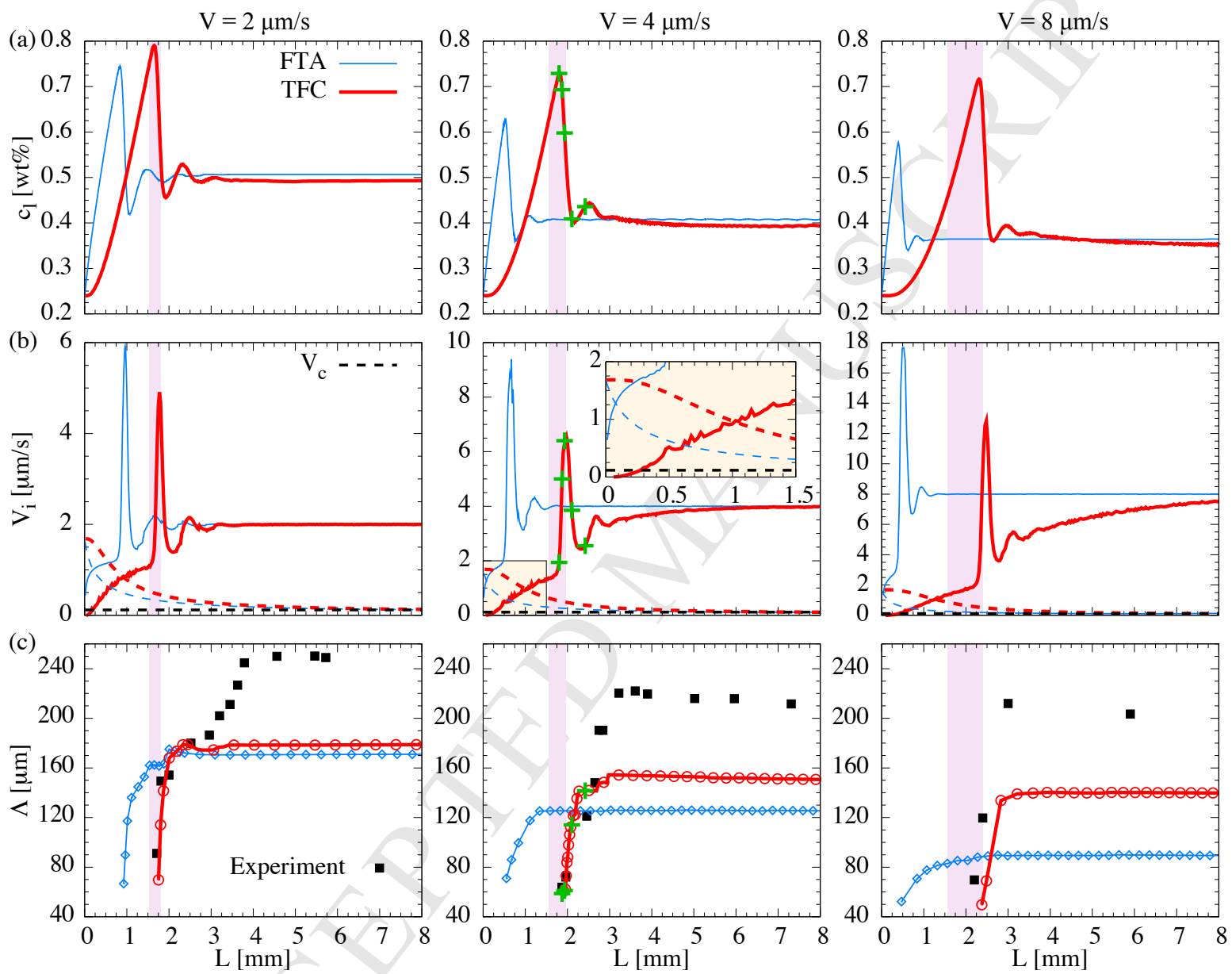

Figure 6: Time evolution of the interface liquid solute concentration $c_{l}$ (a), interface velocity $V_{i}$ (b), and average spacing (c) as a function of the solidified length $L$ for different pulling velocities $V=2$ (left column), 4 (center column), and $8 \mu \mathrm{m} / \mathrm{s}$ (right column) in both FTA (blue thin line) and TFC (red thick line). The shaded area shows the experimentally observed time range for the onset of morphological instability. Plus symbols mark the time steps for snapshots in Fig. 5. The dashed lines in (b) relate to $V_{c}$ using the constitutional undercooling criterion Eq. (5) [39, 38] (constant black dashed line) and a time dependent $\Delta c_{i}(t)$ from planar front simulations (colored dashed lines). 


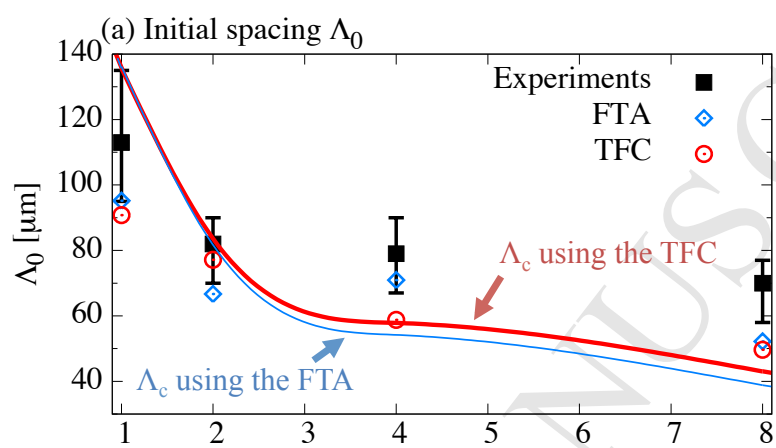

(b) Time for morphological instability

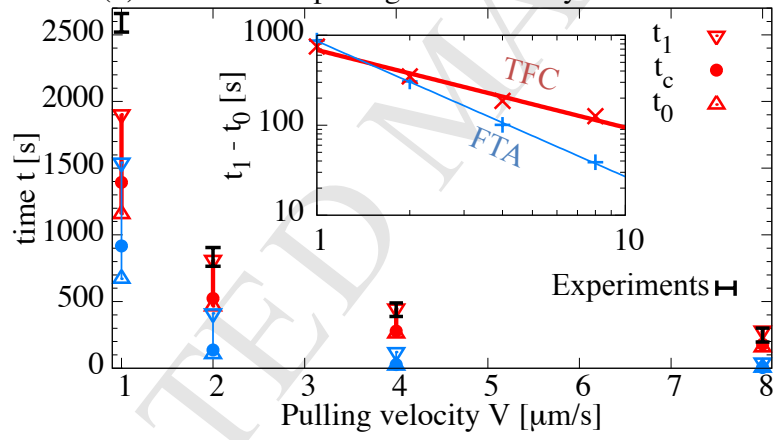

Figure 7: Initially selected spacing $\Lambda_{0}$ (a) and the measured $t_{0}, t_{c}$, and $t_{1}$ (b) as a function of pulling velocity $V$. In (a), measured average initial spacings in the experiments (black squares), and in PF simulations using the FTA (blue diamonds) and the TFC (red circles) are compared to the critical wavelength $\Lambda_{c}$. The black error bars correspond to minimum and maximum spacings in experiments. (b) shows the measured $t_{0}$ (up triangles) when $V_{i}(t)$ intersects $V_{c}(t), t_{c}$ (filled circle dots) the critical time for $\Lambda_{c}$, and $t_{1}$ (down triangles) for the onset of morphological instability from the FTA (blue symbols) and the TFC (red symbols) simulations. Black vertical error bars correspond to time ranges of the morphological instability in the experiments (i.e. red shaded areas in Fig. 4 and Fig. 6). The subset of (b) using a log-log scale shows the time interval between $t_{0}$ and $t_{1}$ in the FTA (blue plus dots) and the TFC (red cross dots) as a function of $V$ with corresponding fitting lines. 

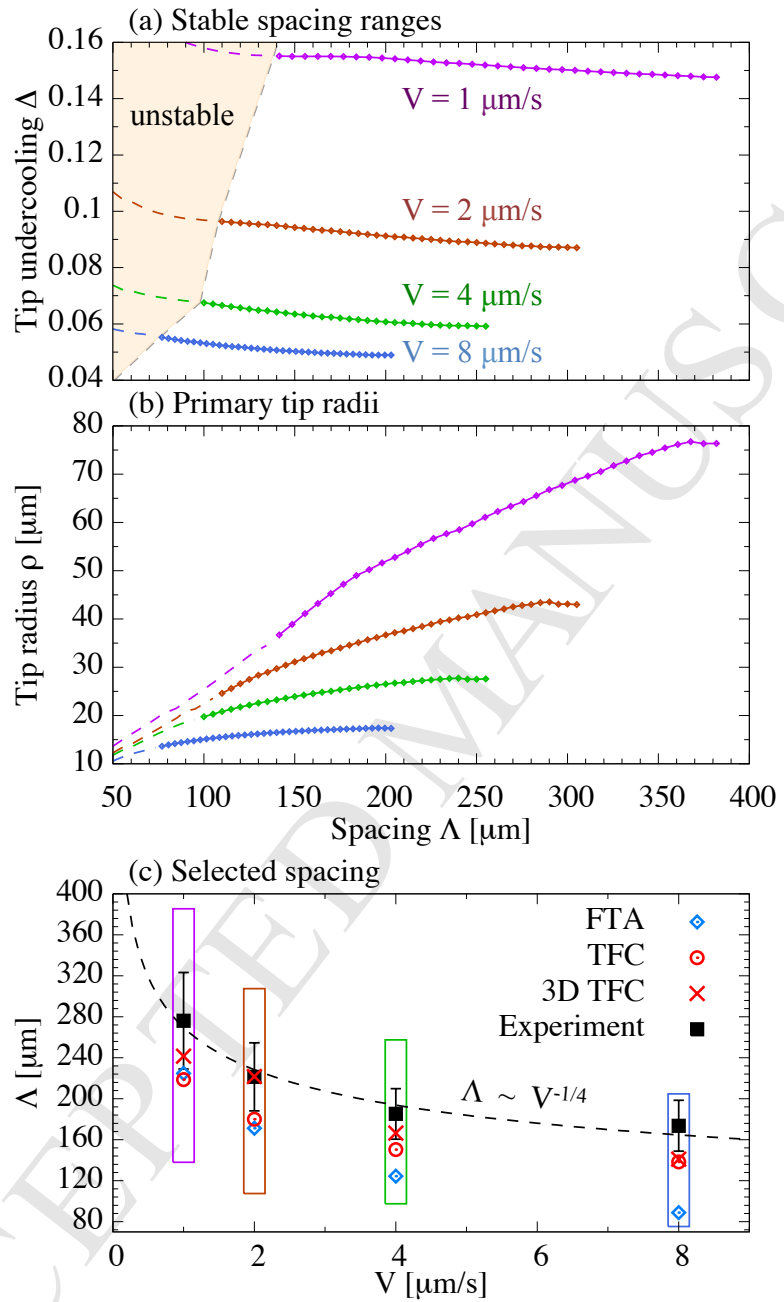

Figure 8: Steady state tip undercooling (a) and radius (b) as a function of the primary spacing, with dashed lines corresponding to spacing unstable to cell elimination. The stable spacing ranges identified for reduced system sizes in (a)-(b) are reported in (c) as rectangular boxes, and compared to dynamically selected spacings in spatially extended domains in experiments (black squares for average and error bars for standard deviations) and in simulations with different thermal representations (color symbols) as a function of the pulling velocity. The black dashed line shows a guideline $\Lambda \sim V^{-1 / 4}$. 


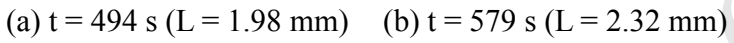

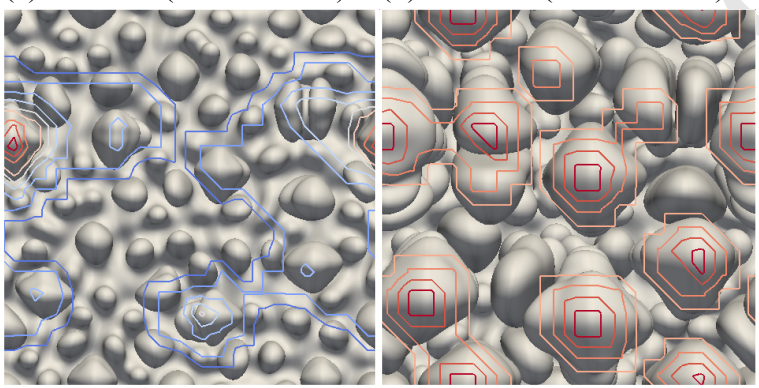

(c) $\mathrm{t}=2011 \mathrm{~s}(\mathrm{~L}=8.04 \mathrm{~mm})(\mathrm{d}) \mathrm{t}=3000 \mathrm{~s}(\mathrm{~L}=12.0 \mathrm{~mm})$

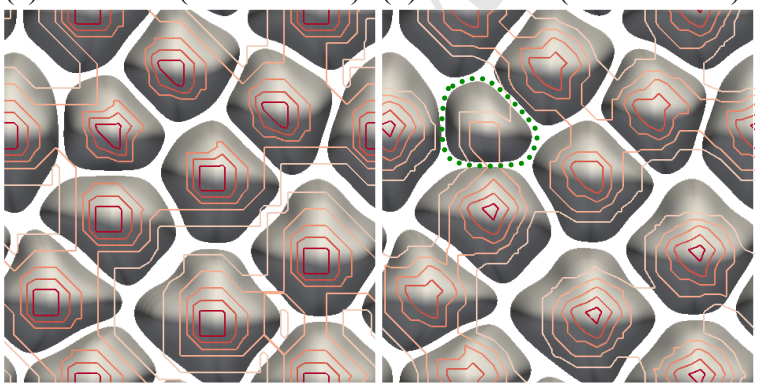

Figure 9: Interface shape and thermal field for $V=4 \mu \mathrm{m} / \mathrm{s}$ at different times $t$ [s] $=$ (a) 494 (near the peak velocity), (b) 579 , (c) 2011, and (d) 3000 (steady state). Contour lines show iso-values of the temperature field on a $z=z_{i}$ plane located just ahead of the most advanced tip from higher temperatures (red) $T_{M a x}[\mathrm{~K}]=$ (a) 330.409, (b) 330.577, (c) 330.617, and (d) 330.623 to lower temperatures (blue) with steps of $0.5 \times 10^{-3} \mathrm{~K}$. Dotted green lines in (d) show the location of a stationary trailing cell. 